\title{
Novel Formulation and Efficient Solution Strategy for Strategic Optimization of an Industrial Chemical Supply Chain Under Demand Uncertainty
}

\author{
Kyle McLean, Emmanuel Ogbe and Xiang Li ${ }^{*}$ \\ Department of Chemical Engineering, Queen's University, 19 Division Street \\ Kingston, ON, K7L 3N6, Canada
}

\begin{abstract}
This paper is concerned with strategic optimization of a typical industrial chemical supply chain, which involves a material purchase and transportation network, several manufacturing plants with on-site material and product inventories, a product transportation network and several regional markets. In order to address large uncertainties in customer demands at the different regional markets, a novel robust scenario formulation, which has been developed by the authors recently, is tailored and applied for the strategic optimization. Case study results show that the robust scenario formulation works well for this real industrial supply chain system, and it outperforms the deterministic formulation and the classical scenario-based stochastic programming formulation by generating better expected economic performance and solutions that are guaranteed to be feasible for all uncertainty realizations. The robust scenario problem exhibits a decomposable structure that can be taken advantage of by Benders decomposition for efficient solution, so the application of Benders decomposition to the solution of the strategic optimization is also discussed. The case study results show that Benders decomposition can reduce the solution time by almost an order of magnitude when the number of scenarios in the problem is large.
\end{abstract}

Keywords: Supply Chain; Uncertainty; Stochastic Programming; Robust Scenario Formulation; Benders Decomposition

\footnotetext{
* Author to whom correspondence may be addressed.

Email address: xiang.li@chee.queensu.ca
} 


\section{Introduction}

Supply chain optimization (SCO) is a set of approaches utilized to efficiently integrate suppliers, manufacturers, warehouses and stores, so that merchandise is produced and distributed at the right quantities, to the right locations, and at the right time, in order to minimize system-wide costs while satisfying service level requirements. ${ }^{[1]}$ SCO has emerged as a major research direction in the process systems engineering (PSE) community since the last decade; in the context of PSE, it is sometimes called enterprise-wide optimization if the emphasis is placed on the manufacturing stage. ${ }^{[2]}$ In the PSE literature, SCO has been intensively studied for a variety of process industries, such as petroleum industry, ${ }^{[3-5]}$ bioenergy industry, ${ }^{[6-8]}$ pharmaceutical industry, ${ }^{[9-10]}$ etc. Papageorgiou gave a comprehensive summary and discussion on the advances and opportunities of supply chain optimization for the process industries. ${ }^{[1]}$ Nikolopoulou and Ierapetritou presented a review on the optimization of sustainable chemical processes and supply chains for balanced economic, environmental and social objectives. ${ }^{[12]}$

SCO problems can be categorized into three levels of problems, namely, strategic, tactical, and operational problems, which are associated with the design, long-term/mid-term planning, and short-term operation of supply chains, respectively. ${ }^{[13]}$ At any decision-making level in SCO, there may be factors that are not known exactly but can significantly impact the supply chain performance, such as those related to raw material supplies, transportation and logistics, production and operation uncertainties, product prices, emissions, etc. Without addressing these factors explicitly, decisions may lead to poor economic or sustainability performance, and even fail to meet stringent customer service levels. 
Among the various approaches to optimization under uncertainty, ${ }^{[14]}$ scenario-based two-stage stochastic programming with recourse ${ }^{[15]}$ is often adopted for SCO under uncertainty. This approach involves a group of first-stage variables representing the decisions made before the realization of uncertainty, a group of second-stage variables representing the decisions made after the realization of uncertainty, and an objective function representing the expected performance (e.g., net present value). The scenario-based approach has limitations in that it cannot guarantee feasibility of its solution for the entire uncertainty region, since usually only a finite subset of uncertainty realizations can be examined. Another classical approach to address uncertainty is a robust approach, ${ }^{[16]}$ which only considers the "worst-case uncertainty realization". On the one hand, this can guarantee that the solution obtained is feasible and overcome the difficulty from the scenario approach. On the other hand, this approach normally leads to conservative solutions with poor expected performance, which is probably why it has not received much attention in the SCO research. ${ }^{[13]}$ Recently, a novel formulation, called robust scenario formulation, has been developed to better address uncertainties in SCO. ${ }^{[17]}$ By combining the notions of the scenario and robust approaches, the robust scenario formulation can achieve a solution that is guaranteed to be feasible (for the bounded uncertainty region considered) and also achieves a good expected economic performance.

Both the scenario formulation and the robust scenario formulation may involve a large number of scenarios needed for effective characterization of uncertainties, leading to a large-scale optimization problem that a regular optimization solver may not be able to solve within a reasonable time. However, both formulations exhibit a special decomposable structure that can be exploited by some decomposition strategies, such as Bender decomposition, ${ }^{[18-19]}$ Dantzig- 
Wolfe decomposition, ${ }^{[20]}$ Lagrangian decomposition, ${ }^{[21]}$. Dantzig-Wolfe decomposition and Lagrangian decomposition cannot guarantee a global solution for mixed-integer optimization unless a branch-and-bound framework is adopted, ${ }^{[21-23]}$ because both of the decomposition strategies rely on the strong duality that is usually lost when integer variables are included in the problem. Benders decomposition, on the other hand, can still guarantee a global solution if integer variables only appear as first-stage decision variables.

This paper aims at strategic optimization of an industrial chemical supply chain from one of the major chemical companies. The supply chain involves significant demand uncertainties, and addressing these uncertainties explicitly is very important for the strategic optimization, as the decisions to be made involve large investment costs and they can influence the whole life cycle of the supply chain. Through this real industrial supply chain system, we demonstrate that the robust scenario formulation leads to solutions with guaranteed feasibility and improved optimality compared to the classical scenario formulation. We also show that the robust scenario formulation retains a decomposable structure that can be exploited by Benders decomposition. The remaining part of the paper is organized as follows. In Section 2, the industrial supply chain system is introduced and a strategic optimization formulation is developed without the consideration of uncertainty. In Section 3, the classical scenario formulation and the robust scenario formulation are introduced, and how the demand uncertainties are modeled is explained. In Section 4, the decomposable structure of the robust scenario formulation and Benders decomposition method that can exploit this structure are discussed. The benefits of the robust scenario formulation and Benders decomposition are demonstrated through case studies in Section 5, and the paper ends with concluding remarks in Section 6. 


\section{The Industrial Chemical Supply Chain}

Figure 1 illustrates the structure of the supply chain. It involves 55 grades of Primary Raw Material (PRM) which when purchased, can either be transported to the PRM warehouse or to any of the 5 on-site PRM warehouses. From the on-site warehouses, the raw material is then processed in one of the 5 manufacturing plants and then sent to the on-site final product warehouses. The final products (FP), which are classified under one of the appropriate 23 possible FP grades, can be transported to regional warehouses for additional storage or to the 5 regional markets to be sold to the end users.

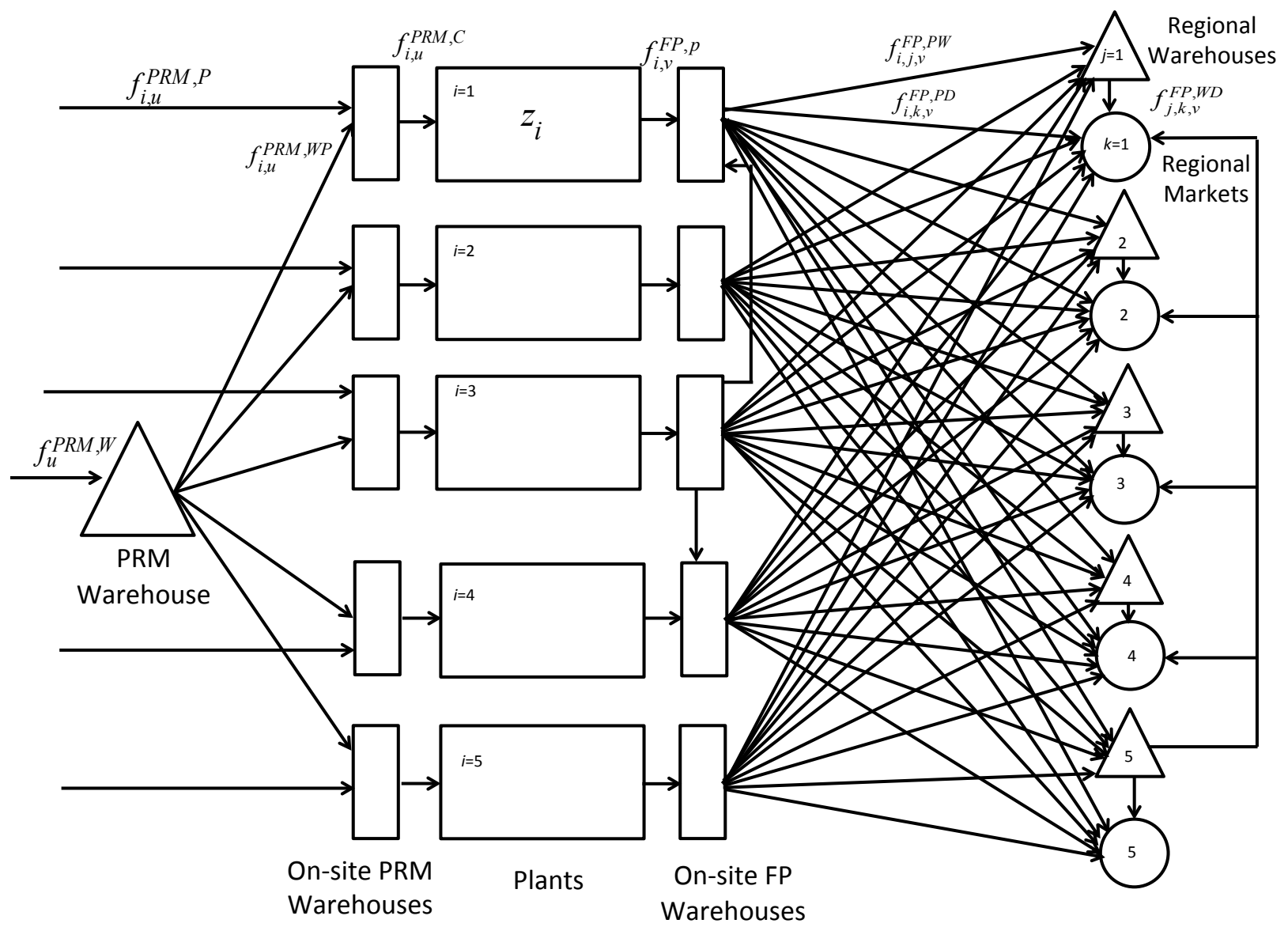

Note: Subscript $u$ indexes 55 grades of PRM and $v$ indexes 23 grades of FP.

Figure 1. Diagram of the industrial chemical supply chain 
The goal of this industrial strategic SCO problem is to determine the optimal capacities for the 5 plants, such that the total profits are maximized and the customer demands at the 5 regional markets are satisfied. Next, we give the deterministic optimization formulation that does not consider the uncertainties. The variables and parameters in the model are listed in the nomenclature at the end of the paper. The values of the parameters are given in the supplementary material.

The objective function to be maximized is the total profit

$$
\sum_{i \in I}\left(\text { Revenue }_{i}-\mathrm{TC}_{i}\right)\left(1-r_{i}^{\mathrm{inc}}\right),
$$

where Revenue ${ }_{i}$ denotes the revenue of plant $i$, calculated from the FP flow rates from the plants to the regional markets $\left(f_{i, k, v}^{\mathrm{FP}, \mathrm{PD}}\right)$ and the flow rates from the plants to the regional warehouses $\left(f_{i, j, v}^{\mathrm{FP}, \mathrm{PW}}\right)$, as follows:

$$
\text { Revenue }_{i}=\sum_{(i, k) \in \Theta} \sum_{v \in V}\left(f_{i, k, v}^{\mathrm{FP}, \mathrm{PD}} \cdot P_{k, v}^{\mathrm{FP}}\right)+\sum_{(i, j) \in \Omega} \sum_{v \in V}\left(f_{i, j, v}^{\mathrm{FP}, \mathrm{PW}} \cdot \hat{P}_{j, v}^{\mathrm{FP}}\right), \quad i \in I .
$$

$\mathrm{TC}_{i}$ denotes the total costs associated with each plant $i$, calculated as the sum of different individual costs as follows:

$$
\mathrm{TC}_{i}=c_{i}^{\mathrm{fix}}+c_{i}^{\mathrm{cap}}+c_{i}^{\mathrm{fr}}+c_{i}^{\mathrm{du}}+c_{i}^{\mathrm{I}}+c_{i}^{\mathrm{PRM}}+c_{i}^{\mathrm{RM} 2}+c_{i}^{\mathrm{RM} 3}+c_{i}^{\mathrm{waste}}+c_{i}^{\mathrm{OPVC}}, \quad i \in I .
$$

Here $c_{i}^{\text {fix }}$ denotes the fixed investment cost for plant $i$, and $c_{i}^{\text {cap }}$ the variable investment cost that is dependent on the capacity of the plant, $z_{i}$. Specifically,

$$
c_{i}^{\text {cap }}=z_{i} C^{\text {cap }}, \quad i \in I,
$$


where $C^{\text {cap }}$ stands for the investment cost per unit capacity. $c_{i}^{\mathrm{fr}}, c_{i}^{\mathrm{du}}, c_{i}^{\mathrm{I}}$ denote the total freight costs, the total duty costs, and the total inventory costs for products produced in plant $i$, respectively. They are calculated based off of the products in the supply chain network that are originally shipped out of the plant, as described by the following equations:

$$
\begin{aligned}
c_{i}^{\mathrm{fr}}= & \sum_{(i, k) \in \Theta} \sum_{v \in V}\left(f_{i, k, v}^{\mathrm{FP}, \mathrm{PD}} C_{i, k}^{\mathrm{ff}, \mathrm{PP}, \mathrm{PD}}\right)+\sum_{(i, j) \in \Omega} \sum_{v \in V}\left(f_{i, j, v}^{\mathrm{FP}, \mathrm{PW}} C_{i, j}^{\mathrm{ff}, \mathrm{FP}, \mathrm{PW}}\right) \\
& +\sum_{(i, j) \in \Omega} \sum_{(j, k) \in \Pi} \sum_{v \in V}\left(f_{j, k, v}^{\mathrm{FP}, \mathrm{WD}} C_{j, k}^{\mathrm{f}, \mathrm{FP}, \mathrm{WD}} q_{i, j}^{\mathrm{fr}, \mathrm{PW}}\right), \quad i \in I, \\
c_{i}^{\mathrm{du}}= & r^{\mathrm{tp}} \cdot\left[\sum_{(i, k) \in \Theta} \sum_{v \in V}\left(f_{i, k, v}^{\mathrm{FP}, \mathrm{PD}} P_{k, v}^{\mathrm{FP}} r_{i, k}^{\mathrm{du}}\right)+\sum_{(i, j) \in \Omega} \sum_{v \in V}\left(f_{i, j, v}^{\mathrm{FP}, \mathrm{PW}} \hat{P}_{j, v}^{\mathrm{FP}} \hat{r}_{i, j}^{\mathrm{du}}\right)\right], \quad i \in I, \\
c_{i}^{\mathrm{I}}= & \sum_{(i, v) \in \Psi}\left(f_{i, v}^{\mathrm{FP}, \mathrm{p}} C_{i}^{\mathrm{PI}}\right)+\sum_{(i, j) \in \Omega} \sum_{v \in V}\left(f_{i, j, v}^{\mathrm{FP}, \mathrm{PW}} C_{j}^{\mathrm{WI}}\right), \quad i \in I,
\end{aligned}
$$

where parameters $C_{i, k}^{\mathrm{ff}, \mathrm{FP}, \mathrm{PD}}, C_{i, j}^{\mathrm{f}, \mathrm{FP}, \mathrm{PW}}, C_{j, k}^{\mathrm{ff,FP}, \mathrm{WD}}, C_{i}^{\mathrm{PI}}, C_{j}^{\mathrm{WI}}, P_{k, v}^{\mathrm{FP}}, \hat{P}_{j, v}^{\mathrm{FP}}$ represent different unit costs and prices, $q_{i, j}^{\mathrm{frPW}}$ denotes the proportion of FP shipped from plant $i$ to regional warehouse $j, r_{i, k}^{\mathrm{du}}$ and $\hat{r}_{i, j}^{\mathrm{du}}$ denote duty rates. The costs of PRM, the other two major raw materials (denoted by RM2 and RM3), wastes, and other plant variable costs, are calculated as follows:

$$
\begin{aligned}
& c_{i}^{\mathrm{PRM}}=\sum_{u \in U}\left(f_{i, u}^{\mathrm{PRM}, \mathrm{c}} C_{i, u}^{\mathrm{PRM}}\right), \quad i \in I, \\
& c_{i}^{\mathrm{RM} 2}=\sum_{u \in U}\left(f_{i, u}^{\mathrm{PRM}, \mathrm{c}} q_{i, u}^{\mathrm{RM} 2}\right) C_{i}^{\mathrm{RM} 2}, \quad i \in I, \\
& c_{i}^{\mathrm{RM} 3}=\sum_{u \in U}\left(f_{i, u}^{\mathrm{PRM}, \mathrm{c}} q_{i, u}^{\mathrm{RM} 3}\right) C_{i}^{\mathrm{RM} 3}, \quad i \in I,
\end{aligned}
$$




$$
\begin{aligned}
& c_{i}^{\mathrm{waste}}=\sum_{u \in U}\left(f_{i, u}^{\mathrm{PRM}, \mathrm{c}} q_{u}^{\text {waste }}\right) C_{i}^{\text {waste }}, \quad i \in I, \\
& c_{i}^{\mathrm{OPVC}}=C_{i}^{\mathrm{var}}\left(\sum_{(i, v) \in \Psi} f_{i, v}^{\mathrm{FP}, \mathrm{p}}\right), \quad i \in I,
\end{aligned}
$$

Where the parameters are explained in the nomenclature.

The materials consumed and the products produced in the manufacturing plants are limited by the following constraints:

$$
\begin{aligned}
& \sum_{u \in U} f_{i, u}^{\mathrm{PRM}, \mathrm{c}} \leq \sum_{(i, v) \in \Psi}\left(\eta_{i}^{t d, 1} f_{i, v}^{\mathrm{FP}, \mathrm{p}}+\eta_{i}^{t d, 2}\right), \quad i \in I, \\
& \sum_{u \in U} f_{i, u}^{\mathrm{PRM}, \mathrm{c}} \leq \sum_{(i, v) \in \Psi}\left(\eta_{i}^{w l, 1} f_{i, v}^{\mathrm{FP}, \mathrm{p}}+\eta_{i}^{w l, 2}\right), \quad i \in I, \\
& \sum_{(i, v) \in \Psi} f_{i, v}^{\mathrm{PP}, \mathrm{p}} \leq z_{i}, \quad i \in I .
\end{aligned}
$$

Eqs. (13-14) mean that the amount of PRM consumed is subject to both a turndown limit and a waste limit at each plant. The right-hand-sides of the equations are regression models for the limits, and the parameters $\eta_{i}^{t d, 1}, \eta_{i}^{t d, 2}, \eta_{i}^{w l, 1}, \eta_{i}^{w l, 2}$ are estimated empirically from plant data. Eq. (15) denotes that the FP produced at each plant is limited by the capacity of the plant. In addition, the capacity of plant $i$ cannot exceed a maximum possible amount $\left(Z_{i}^{\max }\right)$, so

$$
z_{i} \leq Z_{i}^{\max }, \quad i \in I,
$$

The following equation relates the amount of PRM consumed and the amount of FP produced at each plant:

$$
\sum_{u \in U}\left(f_{i, u}^{\mathrm{PRM}, \mathrm{c}} E_{u}^{\mathrm{PRM}}\right) a_{i}^{\mathrm{avg}, \mathrm{FP}} Y_{i}^{\mathrm{FP}}=\sum_{(i, v) \in \Psi} f_{i, v}^{\mathrm{FP}, \mathrm{p}}, \quad i \in I,
$$


where the parameters $E_{u}^{\mathrm{PRM}}, a_{i}^{\text {avg,FP }}, Y_{i}^{\mathrm{FP}}$ are provided by our industrial collaborator, originally estimated from plant operating data. The material and product flows in the supply chain system also need to satisfy a set of constraints from mass balances, inventory limits, material availability, and customer demands. Specifically, the PRM purchased cannot be greater than the PRM availability

$$
\sum_{i \in I} f_{i, u}^{\mathrm{PRM}, \mathrm{P}}+f_{u}^{\mathrm{PRM}, \mathrm{W}} \leq M_{u}^{\mathrm{PRM}}, \quad u \in U
$$

The PRM ending inventory cannot fall below the target inventory at the PRM warehouse

$$
X_{u}^{\mathrm{PRM}, \mathrm{W}}+f_{u}^{\mathrm{PRM}, \mathrm{W}}-\sum_{i \in I} f_{i, u}^{\mathrm{PRM}, \mathrm{WP}} \geq R_{u}^{\mathrm{PRM}, \mathrm{W}}, \quad u \in U
$$

and at each of the plants

$$
X_{i, u}^{\mathrm{PRM}, \mathrm{P}}+f_{i, u}^{\mathrm{PRM}, \mathrm{P}}+f_{i, u}^{\mathrm{PRM}, \mathrm{WP}}-f_{i, u}^{\mathrm{PRM}, \mathrm{c}} \geq R_{i, u}^{\mathrm{PRM}, \mathrm{P}}, \quad i \in I, \quad u \in U .
$$

The ending FP inventory cannot be less than the target inventory at the plants

$$
X_{i, v}^{\mathrm{FP}, \mathrm{P}}+f_{i, v}^{\mathrm{FP}, \mathrm{p}}-\sum_{(i, j) \in \Omega} f_{i, j, v}^{\mathrm{FP}, \mathrm{PW}}-\sum_{(i, k) \in \Theta} f_{i, k, v}^{\mathrm{FP}, \mathrm{PD}} \geq D_{i, v}^{\mathrm{P}} \cdot R_{i, v}^{\mathrm{FP}} / 365, \quad(i, v) \in \Psi,
$$

and at the regional warehouses

$$
X_{j, v}^{\mathrm{PP}, \mathrm{W}}+\sum_{i \in I} f_{i, j, v}^{\mathrm{FP}, \mathrm{PW}}-\sum_{(j, k) \in \Pi} f_{j, k, v}^{\mathrm{FP}, \mathrm{WD}} \geq D_{j, v}^{W} \cdot R_{j, v}^{\mathrm{FP}} / 365, \quad j \in J, v \in V
$$

The FP shipped to the regional markets must meet the minimum demand requirements

$$
\sum_{(i, k) \in \Theta} f_{i, k, v}^{\mathrm{FP}, \mathrm{PD}}+\sum_{(j, k) \in \Pi} f_{j, k, v}^{\mathrm{FP}, \mathrm{WD}} \geq D_{v, k}^{\min }, \quad k \in K, v \in V .
$$


Finally, the PRM purchased from different sources have different impurity contents, and these flows are blended before converted into different grades of FP. In order to ensure the quality of FP, the PRM blends have to satisfy certain impurity specifications, as follows:

$$
\sum_{u \in U}\left(f_{i, u}^{\mathrm{PRM}, \mathrm{c}} q_{u, w}^{\mathrm{imp}}\right) \leq \sum_{u \in U}\left(f_{i, u}^{\mathrm{PRM}, \mathrm{c}} Q_{i, w}^{\mathrm{imp}}\right), \quad i \in I, w \in W
$$

where $q_{u, w}^{\text {imp }}$ denotes the percentage of impurity $w$ in PRM $u$, and $Q_{i, w}^{\text {imp }}$ denotes the maximum allowed percentage of impurity $w$ in the PRM blends plant $i$. In addition, the ratio of the total effective parts of the PRM blends ( $E_{u}^{\mathrm{PRM}}$ ) has to be below a threshold $\left(Q_{i}^{\max }\right)$, so

$$
\sum_{u \in U}\left(f_{i, u}^{\mathrm{PRM}, \mathrm{c}} E_{u}^{\mathrm{PRM}}\right) \leq \sum_{u \in U}\left(f_{i, u}^{\mathrm{PRM}, \mathrm{c}} Q_{i}^{\max }\right), \quad i \in I .
$$

Therefore, the deterministic strategic optimization problem can be formulated as:

\section{Problem (DSCO):}

Maximum Objective $=$ Eq (1)

Subject to: Eqs. (2-25),

All flow rates and capacities are nonnegative.

The material and product flow rates and plant capacities are decision variables, and the optimization problem is a linear programming (LP) problem. In the next section, we discuss how to extend this deterministic formulation to address uncertainties using a robust scenario approach. 


\section{The Robust Scenario Formulation for Demand Uncertainties}

In this paper, we consider the uncertainty in the minimum demands ( $D_{v, k}^{\min }$ in Eq. (23)) that comes from the need to fulfil contracts and pre-orders. Specifically, assume that the minimum demands satisfy

$$
D_{v, k}^{\min }= \begin{cases}\bar{D}_{v, k}^{\min } \xi^{(1)}, & k=1,2,3 \\ \bar{D}_{v, k}^{\min } \xi^{(2)}, & k=4,5\end{cases}
$$

where $\bar{D}_{v, k}^{\min }$ represents known nominal values of $D_{v, k}^{\min }, \xi^{(1)}$ and $\xi^{(2)}$ are independently and uniformly distributed random variables between $[0.6,1.4]$ and $[0.7,1.3]$. In Subsection 3.1, we give a general introduction to how to address uncertainties through a robust scenario formulation. Then in Subsection 3.2, we explain how to apply the robust scenario formulation for the SCO problem with the demand uncertainty.

\subsection{Scenario Formulation and Robust Scenario Formulation}

For convenience of discussion, we express Problem (DSCO) in the following form:

Problem (DP):

$$
\begin{aligned}
\min _{x \in X, y} & c^{T} x+q^{T} y \\
\text { s.t. } & A x \leq b, \\
& t_{i}^{T} x+w_{i}^{T} y \leq 0, \quad i=1, \cdots, m
\end{aligned}
$$


where vector $x \in X \subset \mathbb{R}^{n_{s}}$ includes the decisions on the plant capacities, and vector $y \in \mathbb{R}^{n_{y}}$ includes all other variables, parameters $A \in \mathbb{R}^{l \times n_{x}}, b \in \mathbb{R}^{l}, c, t_{i} \in \mathbb{R}^{n_{x}}, q, w_{i} \in \mathbb{R}^{n_{y}}$. Note that Problem (DP) does not involve equality constraints and the right-hand-sides of Eq. (28) are all zero, but they do not reduce the generality of the formulation. This is because any equality constraint can be expressed as a pair of inequality constrains. In addition, if the right-hand-side of one constraint in Eq. (28) is not zero, say,

$$
t_{i}^{T} x+w_{i}^{T} y \leq h_{i},
$$

then the constraint can be expressed with an extra scalar variable $z$ as follows:

$$
\begin{aligned}
& t_{i}^{T} x-h_{i} z+w_{i}^{T} y \leq 0, \\
& z=1 .
\end{aligned}
$$

Therefore, Problem (DSCO) can always be expressed in form of Problem (DP).

If there exist uncertain factors that are not known before we made the plant capacity decisions but will be known afterward, e.g., costumer demands, then Problem (DP) will involve uncertain parameters, typically in parameters $q, t_{i}$. In this case, the optimization formulation usually needs to minimize the expected performance and enforces that the constraints are satisfied for all possible realizations of uncertain parameters. This leads to a stochastic programming problem in the following form: ${ }^{[15]}$

\section{Problem (SP):}

$$
\begin{aligned}
& \min _{x \in X} c^{T} x+E_{\xi \in \Xi}[Q(x, \xi)] \\
& \text { s.t. } A x \leq b,
\end{aligned}
$$


where $\xi \in \Xi$ denotes the parameters that are not known exactly when determining $x$ (but will be realized before the determination of $y), Q(x, \xi)=\min \left\{q(\xi)^{T} y:\left(t_{i}(\xi)\right)^{T} x+w_{i}^{T} y \leq 0, i=1, \cdots, m\right\}$ denotes the cost associated with a particular group of decisions $x$ and a particular realization of uncertainties $\xi$. In the stochastic programming literature, $x$ is called first-stage variables, $y$ is called second-stage variables, and the optimization subproblem to be solved for computing $Q(x, \xi)$ is called a recourse problem, Problem (SP) is called a two-stage stochastic programming problem with recourse. Problem (SP) is generally intractable if set $\Xi$ includes an infinite number of uncertainty realizations, as an infinite number of recourse problems are to be solved. Therefore, it is often solved approximately through addressing a finite subset of uncertainty realizations in $\Xi$, e.g., $\left\{\xi_{1}, \cdots \xi_{s}\right\} \subset \Xi$. In this case, each uncertainty realization in $\left\{\xi_{1}, \cdots \xi_{s}\right\}$ is called a scenario, and the approximating problem to be solved is called a scenario problem. Figure 2 (a) illustrates the scenarios in the scenario formulation.

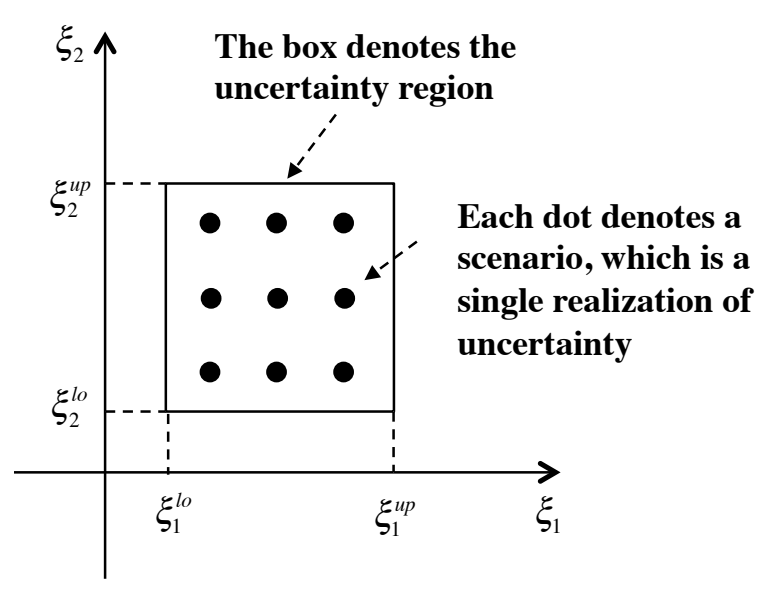

(a) Scenarios in the scenario formulation

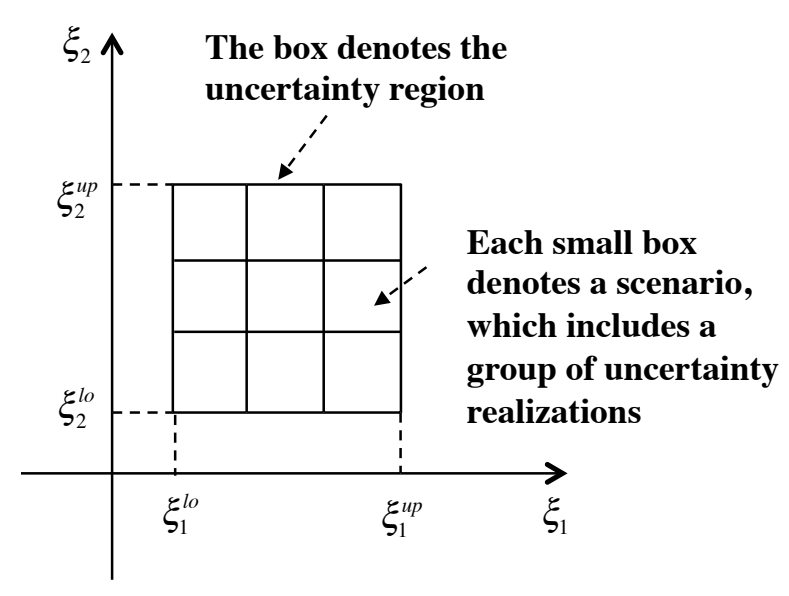

(b) Scenarios in the robust scenario formulation

Figure 2. Comparison of scenarios in the scenario and the robust scenario formulations 
Note that the uncertainty parameters $\xi$ are included in vectors $q$ and $t_{i}$; for convenience of discussion, the values of $q$ and $t_{i}$ in scenario $\omega$ are represented as $q_{\omega}=\alpha_{q} \xi_{\omega}+\beta_{q}$, $t_{i, \omega}=\alpha_{t, i} \xi_{\omega}+\beta_{t, i}$, where $\alpha_{q} \in \mathbb{R}^{n_{y} \times n_{u}}, \beta_{q} \in \mathbb{R}^{n_{y}}, \alpha_{t, i} \in \mathbb{R}^{n_{x} \times n_{u}}, \beta_{t, i} \in \mathbb{R}^{n_{x}}$ are known constants. Thus the scenario formulation can be written in the following form:

\section{Problem (S):}

$$
\begin{aligned}
& \min _{\substack{x \in X, y_{1}, \ldots, y_{s}}} c^{T} x+\sum_{\omega=1}^{s} p_{\omega}\left(\alpha_{q} \xi_{\omega}+\beta_{q}\right)^{T} y_{\omega} \\
& \text { s.t. } A x \leq b, \\
& \qquad\left(\alpha_{t, i} \xi_{\omega}+\beta_{t, i}\right)^{T} x+w_{i}^{T} y_{\omega} \leq 0, \quad i=1, \cdots, m, \omega=1, \cdots, s,
\end{aligned}
$$

where $p_{\omega}$ is the probability of scenario $\omega$, and $y_{\omega}$ is the value of $y$ for scenario $\omega$. With a sufficiently large number of scenarios, Problem (S) can often lead to a solution with a satisfactory expected performance, but it cannot guarantee that the solution is feasible for Problem (SP), because not all realizations of uncertainty are included in Problem (S).

To overcome the problems caused by the scenario formulation, a novel formulation, called robust scenario formulation, has been developed recently to ensure that a solution is feasible for all uncertainty realizations and achieve an improved expected performance. ${ }^{[17]}$ In a robust scenario formulation, a scenario is not a single uncertainty realization, but a subset of uncertainty realizations $\Xi_{\omega}(\omega=1, \cdots, s)$ that includes a (finite or infinite) number of uncertainty realizations. If $\bigcup_{\omega=1}^{s} \Xi_{\omega} \supset \Xi$, then all uncertainty realizations can be addressed through the $s$ scenarios. For each scenario, a robust recourse problem is considered, which ensures feasibility 
for all uncertainty realizations in this scenario through the notion of robust optimization. ${ }^{[16]}$ Figure 2 (b) illustrates the scenarios in the robust scenario formulation.

Two types of robust scenario formulation were developed in the authors' previous work. ${ }^{[17]}$ One is called naïve robust scenario formulation, which only allows one group of second-stage decisions for all realizations of uncertainties with a scenario. The other is called affinely adjustable robust scenario formulation, which allows the second-stage decisions to vary affinely with respect to the uncertainty realizations within a scenario. It has been shown that affinely robust scenario formulation is significantly better than the naïve robust scenario formulation, so this paper only considers this formulation. In this formulation, the second-stage decision for scenario $\omega, y_{\omega}$, is not a single value but an affine function of $\xi_{\omega}$. It is expressed as $U_{\omega} \xi_{\omega}+v_{\omega}$, where $U_{\omega}, v_{\omega}$ are second-stage decision variables for the optimization. Note that the true relationship between $y_{\omega}$ and $\xi_{\omega}$ is not known a priori. The affine function is an approximation of the relationship. The approximation leads to a formulation that underestimates the true expected performance, but it does not affect the feasibility of solution for all uncertainty realizations. ${ }^{[16-17]}$ The affinely robust scenario formulation for Problem (SP) can be written in the following form:

$$
\begin{aligned}
\min _{\substack{x \in X, U_{1}, \cdots U_{s}, v_{1}, \cdots, v_{s}}} c^{T} x+\sum_{\omega=1}^{s} p_{\omega}\left(\alpha_{q} \bar{\xi}_{\omega}+\beta_{q}\right)^{T}\left(U_{\omega} \bar{\xi}_{\omega}+v_{\omega}\right) \\
\text { s.t. } A x \leq b, \\
\max _{\xi_{\omega} \in \Xi_{\omega}}\left\{\left(\alpha_{t, i} \xi_{\omega}+\beta_{t, i}\right)^{T} x+w_{i}^{T}\left(U_{\omega} \xi_{\omega}+v_{\omega}\right)\right\} \leq 0, \quad i=1, \ldots, m, \quad \omega=1, \ldots, S
\end{aligned}
$$


where $\bar{\xi}_{\omega}$ denotes the expected value of $\xi_{\omega}$ for scenario $\omega$, Eq. (36) includes the robust counterparts of inequalities in Eq. (33), $\Xi_{\omega}$ denotes the uncertainty set for the uncertain parameters for scenario $\omega$. If $\Xi_{\omega}$ is a symmetric polyhedral set that can be expressed as $\Xi_{\omega}=\left\{\xi_{\omega}:\left\|M_{\omega}\left(\xi_{\omega}-\bar{\xi}_{\omega}\right)\right\|_{\infty} \leq \delta_{\omega}\right\}$ (where $M_{\omega}, \delta_{\omega}$ are constants determining the shape and size of the polyhedral set), then the left-hand-side of Eq. (36) can be reformulated as ${ }^{[24]}$

$$
\begin{aligned}
& \max _{\xi_{\omega} \in \Xi_{\omega}}\left\{\left(\alpha_{t, i} \xi_{\omega}+\beta_{t, i}\right)^{T} x+w_{i}^{T}\left(U_{\omega} \xi_{\omega}+v_{\omega}\right)\right\} \\
= & \beta_{t, i}^{T} x+w_{i}^{T} v_{\omega}+\left(x^{T} \alpha_{t, i}+w_{i}^{T} U_{\omega}\right) \bar{\xi}_{\omega}+\max _{\xi_{\omega} \in \bar{\sigma}_{\omega}}\left\{\left(x^{T} \alpha_{t, i}+w_{i}^{T} U_{\omega}\right)\left(\xi_{\omega}-\bar{\xi}_{\omega}\right)\right\} \\
= & \beta_{t, i}^{T} x+w_{i}^{T} v_{\omega}+\left(x^{T} \alpha_{t, i}+w_{i}^{T} U_{\omega}\right) \bar{\xi}_{\omega}+\delta_{\omega}\left\|\left(x^{T} \alpha_{t, i}+w_{i}^{T} U_{\omega}\right) M_{\omega}^{-1}\right\|_{1},
\end{aligned}
$$

and the affinely robust scenario formulation can be written as:

\section{Problem (AARS_IN):}

$$
\begin{aligned}
& \min _{\substack{x \in X, U_{1}, \cdots U_{s}, v_{1}, \cdots, v_{s}}} c^{T} x+\sum_{\omega=1}^{s} p_{\omega}\left(\alpha^{(q)} \bar{\varepsilon}_{\omega}+\beta^{(q)}\right)^{T}\left(U_{\omega} \bar{\xi}_{\omega}+v_{\omega}\right) \\
& \text { s.t. } \quad A x \leq b,
\end{aligned}
$$

$$
\begin{array}{r}
\beta_{t, i}^{T} x+w_{i}^{T} v_{\omega}+\left(x^{T} \alpha_{t, i}+w_{i}^{T} U_{\omega}\right) \bar{\xi}_{\omega}+\delta_{\omega}\left\|\left(x^{T} \alpha_{t, i}+w_{i}^{T} U_{\omega}\right) M_{\omega}^{-1}\right\|_{1} \leq 0 \\
i=1, \ldots, m, \omega=1, \ldots, s
\end{array}
$$

The constraints involving 1-norm functions can be transformed into a set of linear constraints, ${ }^{[17]}$ so Problem (AARS_IN) can be transformed into a LP or a mixed-integer linear programming (MILP) problem. Note that when uncertain linear equality constraints are included in Problem (DP), each of them can be transformed into two groups of deterministic equality constraints. See Appendix A for more details. 


\subsection{Expressing the Demand Uncertainty in the Robust Scenario Formulation}

When the uncertainties in $D_{v, k}^{\min }$ are to addressed through the robust scenario approach, Eq. (23) in Problem (DSCO) will be changed into

$$
\begin{array}{r}
\sum_{(i, k) \in \Theta}\left(\Phi_{i, k, v, \omega}^{\mathrm{FP}, \mathrm{PD}} \xi_{\omega}+\phi_{i, k, v, \omega}^{\mathrm{FP}, \mathrm{PD}}\right)+\sum_{(j, k) \in \Pi}\left(\Phi_{j, k, v, \omega}^{\mathrm{FP}, \mathrm{WD}} \xi_{\omega}+\phi_{j, k, v, \omega}^{\mathrm{FP}, \mathrm{WD}}\right) \geq \bar{D}_{v, k}^{\mathrm{min}} \gamma_{k} \xi_{\omega}, \\
k \in K, v \in V, \forall \xi_{\omega} \in \Xi_{\omega}, \omega=1, \cdots, s,
\end{array}
$$

where $\xi_{\omega}=\left(\xi_{\omega}^{(1)}, \xi_{\omega}^{(2)}\right)$ denote realizations of uncertainties in scenario $\omega$ (that is characterized by uncertainty subset $\left.\Xi_{\omega}\right), \Phi_{i, k, v, \omega}^{\mathrm{FP}, \mathrm{PD}} \xi_{\omega}+\phi_{i, k, v, \omega}^{\mathrm{FP}, \mathrm{PD}}$ and $\Phi_{j, k, v, \omega}^{\mathrm{FP}, \mathrm{WD}} \xi_{\omega}+\phi_{j, k, v, \omega}^{\mathrm{FP}, \mathrm{WD}}$ denote the product flow rates that are assumed to vary affinely with $\xi_{\omega}$, and the affine coefficients $\Phi_{i, k, v, \omega}^{\mathrm{FP}, \mathrm{PD}}, \Phi_{j, k, v, \omega}^{\mathrm{FP}, \mathrm{WD}} \in \mathbb{R}^{n_{u}}$, $\phi_{i, k, v, \omega}^{\mathrm{FP}, \mathrm{PD}}, \phi_{j, k, v, \omega}^{\mathrm{FP}, \mathrm{WD}} \in \mathbb{R}$ are decision variables in the robust scenario formulation. $\gamma_{k}=(1,0)$ for $k=1,2,3$, and $\gamma_{k}=(0,1)$ for $k=4,5$.

Now we demonstrate how uncertainty subset $\Xi_{\omega}$ for each scenario is constructed. Since $\xi=\left(\xi^{(1)}, \xi^{(2)}\right) \in[0.6,1.4] \times[0.7,1.3]$, we have

$$
\left|\frac{\xi^{(1)}-1}{0.4}\right| \leq 1 \text { and }\left|\frac{\xi^{(2)}-1}{0.3}\right| \leq 1
$$

or

$$
\left\|\left(\begin{array}{c}
\frac{\xi^{(1)}-1}{0.4} \\
\frac{\xi^{(2)}-1}{0.3}
\end{array}\right)\right\|_{\infty}=\max \left\{\left|\frac{\xi^{(1)}-1}{0.4}\right|, \quad\left|\frac{\xi^{(2)}-1}{0.3}\right|\right\} \leq 1
$$




$$
\left\|\left(\begin{array}{cc}
0.4^{-1} & \\
& 0.3^{-1}
\end{array}\right)\left[\left(\begin{array}{l}
\xi^{(1)} \\
\xi^{(2)}
\end{array}\right)-\left(\begin{array}{l}
1 \\
1
\end{array}\right)\right]\right\|_{\infty} \leq 1 .
$$

So the uncertainty set for the demands can represented by $\Xi=\left\{\xi:\|M(\xi-\bar{\xi})\|_{\infty} \leq \delta\right\}$, where

$$
\bar{\xi}=(1,1), M=\left(\begin{array}{cc}
0.4^{-1} & \\
& 0.3^{-1}
\end{array}\right), \delta=1 .
$$

In the robust scenario formulation, $\Xi$ can be partitioned into $s$ identical subsets in the form of $\Xi_{\omega}=\left\{\xi_{\omega}:\left\|M_{\omega}\left(\xi-\bar{\xi}_{\omega}\right)\right\|_{\infty} \leq \delta_{\omega}\right\}$. For example, if $\Xi$ is partitioned into 9 identical subsets as shown in Figure $2(\mathrm{~b})$, then $\bar{\xi}_{\omega}$ will be the center of each subset and $\delta_{\omega}=1 / 3$ for all subsets. As a result, Eq. (40) can be written in the following form, using the procedure explained in the previous subsection:

$$
\begin{aligned}
& \sum_{(i, k) \in \Theta} \phi_{i, k, v, \omega}^{\mathrm{FP} P \mathrm{PD}}+\sum_{(j, k) \in \Pi} \phi_{j, k, v, \omega}^{\mathrm{FP}, \mathrm{wD}}+\left(\sum_{(i, k) \in \Theta} \Phi_{i, k, v, \omega}^{\mathrm{FP}, \mathrm{PD}}+\sum_{(j, k) \in \Pi} \Phi_{j, k, v, \omega}^{\mathrm{FP}, \mathrm{wD}}-\bar{D}_{v, k}^{\mathrm{min}} \gamma_{k}\right) \bar{\xi}_{\omega} \\
& -\delta_{\omega}\left\|\left(\sum_{(i, k) \in \Theta} \Phi_{i, k, v, \omega}^{\mathrm{FP}, \mathrm{PD}}+\sum_{(j, k) \in \Pi} \Phi_{j, k, v, \omega}^{\mathrm{FP}, \mathrm{wD}}-\bar{D}_{v, k}^{\min } \gamma_{k}\right) M^{-1}\right\| \geq 0, \quad k \in K, v \in V, \omega=1, \cdots, s .
\end{aligned}
$$

The other constraints in Problem (DSCO) can be changed for constructing the robust scenario formulation in a similar way. The overall robust scenario formulation for the supply chain optimization problem is given in Appendix B. In addition, a simple optimization problem is used in Appendix $\mathrm{C}$ to demonstrate the whole procedure of constructing the robust scenario formulation. 


\section{Benders Decomposition for the SCO Problem Under Uncertainty}

Either the scenario formulation (S) or the robust scenario formulation (AARS_IN) can be difficult to solve if a large number of scenarios are needed for characterizing the uncertainties. However, both formulations have a decomposable structure, for supply chain optimization under uncertainty (and actually for all problems that are formulated into a stochastic program). This structure can be exploited by Benders decomposition for efficient solution. ${ }^{[18]}$ Figure 3 (a) illustrates this decomposable structure. The first row of the figure denotes Eq. (32) in Problem (S) or Eq. (38) in Problem (AARS_IN), which involves variables $x$ only. The other rows of the figure denote Eq. (33) in Problem (S) or Eq. (39) in Problem (AARS_IN) for different scenarios, e.g., the second row denotes the constraints for scenario 1 and it only involves variables $x$ and $y_{1}$. Clearly, when $x$ is known, the problem can be decomposed into $s$ subproblems, each of which is a lot easier to solve. Next, we explain how Benders decomposition can take advantage of this problem feature, and give the Benders decomposition algorithm for the problem.

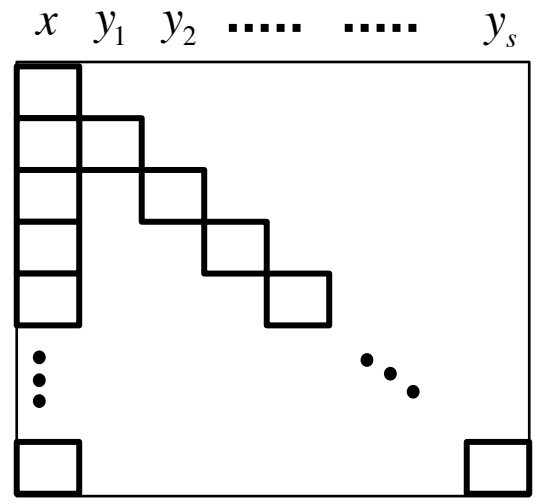

(a) Structure of Problem (S) or (AARS_IN)

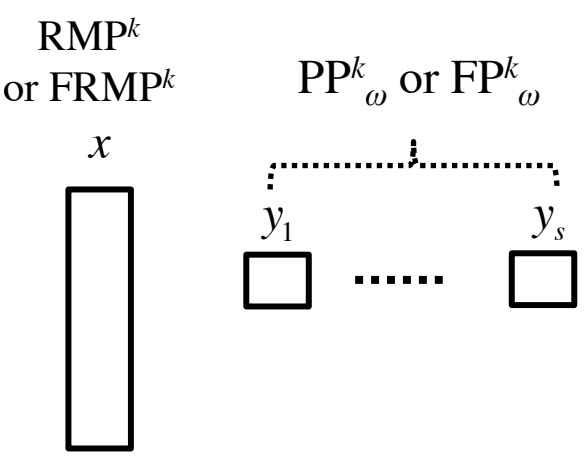

(b) Subproblems in Benders decomposition

Figure 3. The decomposable structure and the Benders decomposition subproblems 
For convenience of discussion, we consider problems in the following form, which covers both Problem (S) and Problem (AARS_IN):

Problem (P):

$$
\begin{array}{ll}
\min _{x, y_{1}, \ldots, y_{s}} & \sum_{\omega=1}^{s}\left(c_{x, \omega}^{T} x+c_{y, \omega}^{T} y_{\omega}\right) \\
\text { s.t. } & A_{\omega} x+B_{\omega} y_{\omega} \leq d_{\omega}, \quad \omega=1, \cdots, s, \\
& x \in X, \\
& y_{\omega} \in Y_{\omega}, \quad \omega=1, \cdots, s,
\end{array}
$$

where $X$ is a compact set, $Y_{\omega}$ is a polyhedral set for all scenarios. At the $k$ th iteration of Benders decomposition, a primal problem is constructed by fixing $x=x^{k}$, and this problem can be decomposed into $s$ subproblems in the following form:

Problem (PP $\left.{ }_{\omega}^{k}\right)$ :

$$
\begin{gathered}
\operatorname{obj}_{P P_{\omega}^{k}}=\min _{y_{\omega}} c_{x, \omega}^{T} x^{k}+c_{y, \omega}^{T} y_{\omega} \\
\text { s.t. } \quad A_{\omega} x^{k}+B_{\omega} y_{\omega} \leq d_{\omega}, \\
y_{\omega} \in Y_{\omega} .
\end{gathered}
$$

Note that Problem $\left(\mathrm{PP}_{\omega}^{k}\right)$ is much easier than Problem $(\mathrm{P})$, because it only involves second-stage decisions for a single scenario and its size is independent of the number of scenarios involved in the original problem. If Problem $\left(\mathrm{PP}_{\omega}^{k}\right)$ is infeasible for $x=x^{k}$, a feasibility problem can be solved, which can be decomposed into s subproblems that are in the following form:

$\operatorname{Problem}\left(\right.$ FP $\left._{\omega}^{k}\right)$ :

$$
\begin{gathered}
o b j_{F P_{\omega}^{k}}=\min _{y_{\omega}, z_{\omega}}\left\|z_{\omega}\right\| \\
\text { s.t. } \quad A_{\omega} x^{k}+B_{\omega} y_{\omega} \leq d_{\omega}+z_{\omega}, \\
y_{\omega} \in Y_{\omega},
\end{gathered}
$$




$$
z_{\omega} \geq 0
$$

where $\|\cdot\|$ denotes an arbitrary norm function. In this paper, the 1-norm is used for all the case studies.

In the initial iteration, $x$ is fixed to an arbitrarily chosen value, but in the subsequent iterations, the value is generated by solving the following relaxed master problem:

\section{Problem $\left(\right.$ RMP $\left.^{k}\right)$ :}

$$
\begin{array}{ll}
\min _{x, \eta} \eta & \\
\text { s.t. } & \eta \geq \sum_{\omega=1}^{s} o b j_{P P_{\omega}^{j}}+\left[\sum_{\omega=1}^{s}\left(c_{x, \omega}^{T}+\left(\lambda_{\omega}^{j}\right)^{T} A_{\omega}\right)\right]\left(x-x^{j}\right), \quad \forall j \in T^{k}, \\
& 0 \geq \sum_{\omega=1}^{s} o b j_{F P_{\omega}^{i}}+\left[\sum_{\omega=1}^{s}\left(\mu_{\omega}^{i}\right)^{T} A_{\omega}\right]\left(x-x^{i}\right), \quad \forall i \in S^{k}, \\
& x \in X,
\end{array}
$$

where the index sets

$$
\begin{aligned}
T^{k} & =\left\{j \in\{1, \ldots, k\}: \text { Problem }(\mathrm{P}) \text { is feasible for } x=x^{j}\right\}, \\
S^{k} & =\left\{i \in\{1, \ldots, k\}: \text { Problem }(\mathrm{P}) \text { is infeasible for } x=x^{i}\right\},
\end{aligned}
$$

$\lambda_{\omega}^{j}$ denotes the Lagrange multipliers for constraint (47) for Problem $\left(\mathrm{PP}_{\omega}^{j}\right)$, and $\mu_{\omega}^{i}$ denotes the Lagrange multipliers for constraint (50) for Problem $\left(\mathrm{FP}_{\omega}^{i}\right)$. Constraints (54) are called optimality cuts, which are obtained via the solutions of the previous solved primal subproblems, and constraints (55) are called feasibility cuts, which are obtained via the solutions of the previously solved feasibility subproblems. Compared to Problem (P), Problem $\left(\mathrm{RMP}^{k}\right)$ is also a much easier problem, as it only involves the first-stage variable $x$ and its size is independent of 
the number of scenarios considered. At the first several iterations, Problem (P) may be infeasible for all the chosen values for $x$, then set $T^{k}$ is empty and Problem $\left(\mathrm{RMP}^{k}\right)$ is unbounded. For these iterations, the following feasibility relaxed master problem is solved instead:

Problem (FRMP $\left.{ }^{k}\right)$ :

$$
\begin{array}{ll}
\min _{x} & \|x\| \\
\text { s.t. } \quad 0 \geq \sum_{\omega=1}^{s} o b j_{F P_{\omega}^{i}}+\left[\sum_{\omega=1}^{s}\left(\mu_{\omega}^{j}\right)^{T} A_{\omega}\right]\left(x-x^{j}\right), \quad \forall i \in S^{k}, \\
\\
x \in X .
\end{array}
$$

Again, $\|\cdot\|$ denotes an arbitrary norm function, and the 1-norm is used for Eq. (57) for all the case studies in this paper.

Benders decomposition solves Problem $\left(\mathrm{PP}_{\omega}^{k}\right)\left(\right.$ or $\left.\left(\mathrm{FP}_{\omega}^{k}\right)\right)$ and Problem $\left(\mathrm{RMP}^{k}\right)\left(\right.$ or $\left.\left(\mathrm{FRMP}^{k}\right)\right)$ iteratively. The solutions of Problem ( $\left.\mathrm{PP}_{\omega}^{k}\right)$ provide a sequence of upper bounds for Problem (P) while the solutions of Problem $\left(\mathrm{RMP}^{k}\right)$ provide a sequence of lower bounds for Problem (P), and an optimal solution is obtained when the upper and lower bounds converge. Figure 3 (b) illustrates the subproblems to be solved in Benders decomposition. The algorithm is described in Figure 4. More discussions on Benders decomposition can be found in the literature. ${ }^{[18-19]}$ 
1. (Initialization) Set upper bound $U B D=M>0$ (which is sufficiently large), lower bound $L B D=-M$, tolerance $\varepsilon>0, k=1, T^{0}=S^{0}=\varnothing$. Select initial values $x_{0}^{1}$.

2. (Primal Problem) Solve Problem $\left(\mathrm{PP}_{\omega}^{k}\right)$ for $\omega=1, \cdots, s$.

3. (Feasible Primal) If Problem $\left(\mathrm{PP}_{\omega}^{k}\right)$ is feasible for $\omega=1, \cdots, s$, an optimal solution $y_{\omega}^{k}$, the associated Lagrange multipliers $\lambda_{\omega}^{k}$ and the optimal objective value $o b j_{P P_{\omega}^{k}}$ can be obtained for $\omega=1, \cdots, s$. Set $T^{k}=T^{k-1} \cup\{k\}, S^{k}=S^{k-1}$, and add an optimality cut to the relaxed master problem. If $\sum_{\omega=1}^{s} o b j_{P P_{\omega}^{k}}<U B D$, set $U B D=\sum_{\omega=1}^{s} o b j_{P P_{\omega}^{k}}$ and $\left(x^{*}, y_{1}^{*}, \cdots y_{s}^{*}\right)=\left(x^{k}, y_{1}^{k}, \cdots y_{s}^{k}\right)$.

4. (Infeasible Primal) If Problem ( $\left.\mathrm{PP}_{\omega}^{k}\right)$ is infeasible for some $\omega$, then solve Problem ( $\left.\mathrm{FP}_{\omega}^{k}\right)$ and obtain Lagrange multipliers $\mu_{\omega}^{k}$ and the optimal objective value $o b j_{F P_{\omega}^{k}}$ for $\omega=1, \cdots, s$. Set $S^{k}=S^{k-1} \cup\{k\}, T^{k}=T^{k-1}$, and add a feasibility cut to the relaxed master problem.

\section{5. (Relaxed Master Problem)}

(a) If $T^{k}=\varnothing$, solve Problem $\left(\mathrm{FRMP}^{k}\right)$. If the problem is feasible and an optimal solution $\hat{x}$ is obtained, set $x^{k+1}=\hat{x}$.

(b) If $T^{k} \neq \varnothing$, solve Problem $\left(\mathrm{RMP}^{k}\right)$. If the problem is feasible and an optimal solution $(\hat{x}, \hat{\eta})$ is obtained, set $x^{k+1}=\hat{x}$ and $L B D=\hat{\eta}$.

5. (Termination Check) If Problem $\left(\mathrm{RMP}^{k}\right)$ or $\left(\mathrm{RMP}^{k}\right)$ is infeasible, terminate and Problem (P) is infeasible. If $U B D \leq L B D+\varepsilon$, terminate and $\left(x^{*}, y_{1}^{*}, \cdots y_{s}^{*}\right)$ is an optimal solution of Problem (P); otherwise, set $k=k+1$ and go to step 2 .

Figure 4. Benders decomposition algorithm for Problem (P) 


\section{Case Studies}

The goals of the case studies are to demonstrate the advantages of the robust scenarios formulation and the computational advantage of Benders decomposition for the industrial SCO problem. All case study problems and the subproblems solved in Benders decomposition were modeled using GAMS 22.1, ${ }^{25}$ and the main body of Benders decomposition was implemented on MATLAB R2013a. ${ }^{26}$ They were all solved using CPLEX $12.5,{ }^{27}$ on a machine with 3.20 GHz CPU, 24 GB RAM and Linux operating system. A relative termination criterion of $10^{-3}$ was used for all the case study problems.

\subsection{Comparison of Three Formulations}

Three optimization formulations are compared here, including the deterministic strategic SCO problem (DP), the scenario problem (S), and the robust scenario problem (AARS_IN). The uncertain parameters $\xi=\left(\xi^{(1)}, \xi^{(2)}\right)$ are treated differently in the three formulations. In Formulation (DP), the expected values of $\xi=\left(\xi^{(1)}, \xi^{(2)}\right)$ are used (i.e., $\left.\xi=(1,1)\right)$. In Formulation (AARS_IN), the ranges of $\xi^{(1)}, \xi^{(2)}$ are evenly partitioned into $n_{s}$ subranges, then the uncertainty region are partitioned into to $n_{s}{ }^{2}$ subregions (which can also be described with the infinity-norm). The mean values of $\xi$ in the subregions are used as the uncertainty realizations addressed in Formulation (S). Note that here we only use simple approaches to generate scenarios for Formulations (AARS_IN) and (S). According to how the robust scenario formulation (AARS_IN) is constructed, the performance of the formulation is not sensitive to how the scenarios (i.e., uncertainty subregions) are generated. On the other hand, the performance of the scenario formulation (S) is highly dependent on how the scenarios (i.e., 
uncertainty realizations) are selected; for the case studies in paper, there may be other scenario generation methods that can help Formulation (S) to yield better prediction of expected profit and solutions that are feasible for all uncertainty realizations, but none of the scenario generation methods can guarantee satisfactory solutions for all stochastic programming problems, especially for those in which the worst-case uncertainty realizations is not obvious.

The three formulations are compared in terms of problems sizes, solutions times (with CPLEX), optimal decisions (i.e., capacities of the plants), optimal objective values, and the actual expected profits achieved by the optimal solution. Here the optimal objective value of a formulation represents the expected profit predicted by the formulation at its optimal solution, which is usually different from the expected profit that can actually be achieved by the optimal solution. The closer the optimal objective value to the actual expected profit is, the better the formulation is in predicting the economic performance and generating more reasonable solutions. Rigorously speaking, the actual expected profit should be obtained by fixing the first-stage decisions to the optimal solution and solving the second-stage recourse problem for all uncertainty realizations. However, when the number of scenarios is infinite, such as in all the problems considered in the case studies, the exact value of the actual expected profit cannot be practically calculated. Therefore, we estimate the actual expected profit using a large number of uncertainty realizations. For all case studies in this paper, 99 values of each of the two independent uncertain parameters were sampled for the estimation of the actual expected profits, using the same approach that is used for the scenario formulation. And this leads to $99^{2}=9801$ uncertainty realizations. 
The three formulations are compared for three cases of the industrial SCO problem. Case A is a base case, where the formulations and parameters used are same to those described in Sections 2 and 3. Table 1 summarizes the results for the three formulations for Case A. It can be seen that Formulation (DP) and Formulation (S) with 9 and 1225 scenarios lead to infeasible capacity decisions, whereas Formulation (AARS_IN) leads to feasible decisions with 9 scenarios (i.e. 9 uncertainty subregions). Formulation (DP) and Formulation (S) give infeasible decisions, because they do not address all possible uncertainty realizations. On the other hand, Formulation (AARS_IN) addresses the feasibility of the solution by considering all possible uncertainty realizations, so it leads to a higher total capacity of the plants, which can hedge against insufficient product supply due to large customer demands. In addition, Formulation (S) leads to a very large optimization problem even when the number of uncertainty realizations addressed is 1225, and this problem took CPLEX 37 minutes to return a solution that is actually infeasible. In contrast, Formulation (AARS_IN) with 9 scenarios has a much smaller problem size, taking CPLEX only 6 minutes to return a feasible and high quality solution.

Case B is same to Case A except that in all formulations, the constraints involving uncertain demands are "softened" with slack variables. These slack variables are penalized in the objective function with a penalty cost $C^{p e n}=200,000 \$ / T$. In this case, minimum customer demands do not need to be all met, so all formulations can give feasible capacity decisions. However, not being able to ensure $100 \%$ demand satisfaction may lead to high penalty costs that can significantly reduce the total profit. Table 2 summarizes the results for Case B. It can be seen that Formulation (DP) gives a very poor solution that actually leads to negative expected profit (although it predicts a very good expected profit). Formulation (S) with 9 scenarios gives a better 
solution, as it considers more realizations of demand uncertainties. However, it still overestimates the expected profit it can achieve. Formulation (AARS_IN) with 9 scenarios gives the best solution, and its prediction of expected profit is very close to the true expected profit. Again, Formulation (AARS_IN) has the best performance as it considers all uncertainty realizations by considering the 9 uncertainty subregions instead of only 9 uncertainty realizations.

Table 1. Solution Results for Case A

\begin{tabular}{|c|c|c|c|c|}
\hline & \multirow{2}{*}{ Formulation (DP) } & \multicolumn{2}{|c|}{ Formulation (S) } & $\begin{array}{c}\text { Formulation } \\
\text { (AARS_IN) }\end{array}$ \\
\hline Number of scenarios $^{\mathrm{a}}$ & 1 & 9 & 1,225 & 9 \\
\hline Number of variables & 2,335 & 20,927 & $2,846,911$ & 73,901 \\
\hline Number of constraints & 1,021 & 9,101 & $1,237,261$ & 132,941 \\
\hline CPLEX solution time (s) & 0.09 & 0.51 & $2,245.53$ & 346.72 \\
\hline & $1-8870$ & $1-7890$ & $1-7940$ & $1-7400$ \\
$2-880$ & $2-1060$ & $2-1160$ & $2-1170$ \\
Plant Capacities at the & $3-440$ & $3-530$ & $3-570$ \\
$4-2120$ & $5-1010$ & $5-1430$ & $4-2270$ \\
optimal solution (10 $\left.{ }^{3} \mathrm{~T}\right)$ & $5-440$ & 21.86 & 21.73 & 21.70 \\
\hline $\begin{array}{c}\text { Optimal objective value } \\
\text { (Billion \$) }\end{array}$ & 22.03 & Infeasible & Infeasible & 21.71 \\
\hline $\begin{array}{c}\text { Actual expected profit } \\
\text { achieved by the optimal } \\
\text { solution (Billion \$) }\end{array}$ & Infeasible & & & \\
\hline
\end{tabular}

Note: ${ }^{\text {a }}$ A scenario means a single uncertainty realization for Formulation (S) and an uncertainty subset for Formulation (AARS_IN). ${ }^{b}$ The actual expected profit is estimated through $99^{2}=9801$ uncertainty realizations. 
Table 2. Solution Results for Case B

\begin{tabular}{|c|c|c|c|}
\hline & Formulation (DP) & Formulation (S) & Formulation (AARS_IN) \\
\hline Number of scenarios $^{\mathrm{a}}$ & 1 & 9 & 9 \\
\hline Number of variables & 2,452 & 21,972 & 77,016 \\
\hline Number of constraints & 1,023 & 9,111 & 137,091 \\
\hline CPLEX solution time (s) & 0.09 & 0.53 & 340.24 \\
\hline & $1-8870$ & $1-7890$ & $1-7400$ \\
Plant Capacities at the & $2-880$ & $2-1060$ & $3-580$ \\
optimal solution (10 3 T) & $3-440$ & $3-530$ & $4-2270$ \\
& $5-2120$ & $4-2240$ & $5-1310$ \\
\hline $\begin{array}{c}\text { Optimal objective value } \\
\text { (Billion \$) }\end{array}$ & 22.03 & $5-1010$ & 21.69 \\
\hline $\begin{array}{c}\text { Actual expected profit } \\
\text { achieved by the optimal } \\
\text { solution (Billion \$) }\end{array}$ & -31.55 & 21.86 & 21.71 \\
\hline
\end{tabular}

Note: ${ }^{a}$ A scenario means a single uncertainty realization for Formulation (S) and an uncertainty subset for Formulation (AARS_IN). ${ }^{b}$ The actual expected profit is estimated through $99^{2}=9801$ uncertainty realizations.

Case $\mathrm{C}$ is another variant of Case $\mathrm{A}$, in which a different set of expected values of $D_{v, k}^{\min }$ (i.e., $\left.\bar{D}_{v, k}^{\min }\right)$ is used. In this case, no feasible capacity decisions exist, as the worst-case customer demands cannot be satisfied even when all plants are developed with their maximum possible capacities. Table 3 summarizes the results for Case $C$, from which it can be seen that Formulations (DP) and (S) cannot correctly indicate the infeasibility of the problem, even when 1225 uncertainty realizations are considered, while Formulation (AARS_IN) indicates the infeasibility with 9 uncertainty subsets. 
Table 3. Solution Results for Case C

\begin{tabular}{|c|c|c|c|c|}
\hline & Formulation (DP) & \multicolumn{2}{|c|}{ Formulation (S) } & Formulation \\
\hline Number of scenarios ${ }^{a}$ & 1 & 9 & 1,225 & 9 \\
\hline Number of variables & 2,335 & 20,927 & $2,846,911$ & 73,901 \\
\hline Number of constraints & 1,021 & 9,101 & $1,237,261$ & 132,941 \\
\hline CPLEX solution time (s) & 0.12 & 0.49 & $2,466.30$ & 433.51 \\
\hline $\begin{array}{l}\text { Plant Capacities at the } \\
\text { optimal solution }\left(10^{3} \mathrm{~T}\right)\end{array}$ & $\begin{array}{l}1-6810 \\
2-1330 \\
3-660 \\
4-2284 \\
5-1630\end{array}$ & $\begin{array}{l}1-5350 \\
2-1600 \\
3-800 \\
4-2600 \\
5-2350\end{array}$ & $\begin{array}{l}1-4670 \\
2-1750 \\
3-870 \\
4-2810 \\
5-2600\end{array}$ & - \\
\hline $\begin{array}{c}\text { Optimal objective value } \\
\text { (Billion \$) }\end{array}$ & 20.97 & 20.47 & 20.19 & $\begin{array}{l}\text { Infeasibility } \\
\text { indicated. }\end{array}$ \\
\hline $\begin{array}{l}\text { Actual expected profit } \\
\text { achieved by the optimal } \\
\text { solution }^{\text {b }} \text { (Billion } \$ \text { ) }\end{array}$ & Infeasible & Infeasible & Infeasible & - \\
\hline
\end{tabular}

Note: ${ }^{a}$ A scenario means a single uncertainty realization for Formulation (S) and an uncertainty subset for Formulation (AARS_IN). ${ }^{b}$ The actual expected profit is estimated through $99^{2}=9801$ uncertainty realizations.

\subsection{Computational Performance of Benders Decomposition}

Case D is studied here to demonstrate the computational advantage of Benders Decomposition. Again, this case is a variant of Case A, where the capacity of each plant cannot be an arbitrary real number but a multiple of $500 \mathrm{~T}$. Therefore, variable $z_{i}$ is replaced by $500 \cdot z_{i}^{\text {int }}$ in the problem and $z_{i}^{\text {int }}$ is a nonnegative integer variable. Note that in this case, the optimization problem is a MILP instead of a LP, no matter which of the three formulations is used. Benders decomposition can still give an optimal solution for the problem, while other classical decomposition methods (such as Dantzig-Wolfe decomposition and Lagrangian decomposition) generally cannot. 
The computational results for Formulation (AARS_IN) with different numbers of scenarios are given in Table 4. In this table, the solution times required by CPLEX to solve the full formulation and the solutions times required by Benders decomposition are compared. It can be seen that CPLEX is faster than Benders decomposition when the number of scenarios is small, but the solution time it requires grows much more quickly with the number of scenarios. As a result, when the number of scenarios is larger than 49 , Benders decomposition starts to be faster than CPLEX. Table 4 also shows the solution times for different Benders subproblems and the total solution time for all Benders subproblems. It can be seen that the solution times for Problems (RMP) and (FRMP) are negligible compared to those for Problems (PP) and (FP). This is because Problems (RMP) and (FRMP) only include the 5 integer decisions, while (PP) and (FP) include all the other variables and the number of (PP) or (FP) subproblems to be solved at each iteration is proportional to the number of scenarios addressed.

Another interesting observation is that the total time for the Benders subproblems is significantly shorter than the overall time for executing Benders decomposition. This significant discrepancy comes from the large computing overhead, mainly due to the slow data exchange between GAMS and MATLAB and partly due to the relatively inefficient programming flow control in MATLAB. This indicates that Bender decomposition could take less time if it had been implemented with a more efficient programming language such as $\mathrm{C} / \mathrm{C}++$. If the computing overhead could be reduced to be negligible, then Benders decomposition could be nearly 10 times faster than CPLEX when the number of scenarios is 169 . 
Table 4. Computational Results for Formulation (AARS_IN) in Case D

\begin{tabular}{|c|c|c|c|c|c|c|c|}
\hline $\begin{array}{c}\text { Number of } \\
\text { Scenarios }\end{array}$ & 1 & 9 & 25 & 49 & 81 & 121 & 169 \\
\hline $\begin{array}{c}\text { CPLEX } \\
\text { solution time (s) }\end{array}$ & 42 & 1400 & 8554 & 27079 & 72245 & 95885 & 281268 \\
\hline $\begin{array}{c}\text { Benders } \\
\text { decomposition } \\
\text { solution time (s) }\end{array}$ & 235 & 2342 & 13065 & 28325 & 30767 & 46863 & 53321 \\
\hline $\begin{array}{c}\text { Total time for } \\
\text { Benders } \\
\text { subproblems (s) }\end{array}$ & 97 & 1278 & 7036 & 17291 & 22371 & 30745 & 35739 \\
\hline $\begin{array}{c}\text { Time for } \\
\text { PP/FP (s) }\end{array}$ & 97 & 1278 & 7036 & 17291 & 22371 & 30745 & 35739 \\
\hline $\begin{array}{c}\text { Time for } \\
\text { RMP/FRMP (s) }\end{array}$ & 0.1 & 0.2 & 0.3 & 0.7 & 0.4 & 0.7 & 0.5 \\
\hline
\end{tabular}

\section{Conclusions}

The industrial chemical SCO problem involves large demand uncertainties that need to be addressed for achieving high profits and customer service levels. The affinely adjustable robust scenario formulation is adopted to maximize the expected total profit while ensuring that the strategic decisions on plant capacities are feasible for all uncertainty realizations. The demand uncertainties are modeled with two independently and uniformly distributed uncertain parameters, and it is shown that the uncertainty subsets of the uncertain parameters can be expressed with the infinity-norm and therefore the robust scenario formulation can lead to a LP or MILP problem. The case study results show that the robust scenario formulation can guarantee the feasibility of its solution or indicate infeasibility when the SCO problem is infeasible, while the deterministic formulation or the scenario formulation cannot. This is because the robust scenario formulation considers all uncertainty realizations by considering a 
finite number of uncertainty subsets, and deterministic or scenario formulation only considers a finite subset of uncertainty realizations. Due to its decomposable structure, the robust scenario problem can be solved by Benders decomposition efficiently, and the case study results demonstrate that Benders decomposition can reduce the solution time by an order of magnitude when the problem includes a large number of scenarios and the computing overhead is minimized. Since any problem that is formulated using a scenario or a robust scenario approach exhibits the same decomposable structure, so Benders decomposition is not limited to supply chain optimization.

The robust scenario formulation has two major advantages. One is that it guarantees the feasibility of the solution for all uncertainty realizations in spite of how the scenarios (i.e., uncertainty subsets) are constructed. This is a significant advantage over the classical scenario formulation, which requires an ideal sampling rule to guarantee the feasibility for all uncertainty realizations and such ideal sampling rule is normally not known for a particular problem. The other advantage is that it approximates the expected economic performance by the weighted average of the "mean performances of each uncertainty subset". This is similar to how the expected performance is estimated in the classical scenario formulation, and it usually provides a satisfactory performance estimate when the number of scenarios is sufficiently large. This is a significant advantage over the classical robust optimization formulation, in which only the nominal or the worst-case economic performance is optimization.

The current robust scenario formulation has some limitations that need to be overcome in the future work. Firstly, it is limited to uncertainty sets that can be expressed with the infinity-norm, 
so it cannot address ellipsoidal or un-symmetric uncertainties sets that are not uncommon in supply chain or process optimization problems. Secondly, with the use of affine approximation of the relationship between uncertainty realizations and second-stage decisions, the formulation overestimates the expected cost (or underestimates the expected profit). Although the gap between the predicted expected cost and the actual one is very small for the case study problems in this paper, it may not be the case for other problems. So it is interesting to find a generic and efficient way to quantify the gap without exhaustive simulation. Finally, the current formulation is developed for problems with linear models. When nonlinear models are present, the formulation is highly nonconvex, and some extension of Benders decomposition is needed for efficient global optimization, such as nonconvex generalized Benders decomposition. ${ }^{[28]}$

\section{Acknowledgements}

The authors are grateful to the financial support from DuPont-Queen's Innovation Programme (DuQuIP). They also thank for Dr. Defne Berkin for help on defining and formulating the supply chain problem.

\section{References}

[1] Simchi-Levi, D.; Kaminsky, P.; Simchi-Levi, E. Designing and managing the supply chain: Concepts, strategies, and cases, McGraw-Hill, 1999.

[2] Grossmann, I. E. Enterprise-wide optimization: A new frontier in process systems engineering. AIChE J. 2005, 51, 1846-1857. 
[3] Neiro, S. M. S.; Pinto, J. M. A general modelling framework for the operational planning of petroleum supply chains. Comput. Chem. Eng. 2004, 28, 871-896.

[4] Schulz, E.P.; Diaz, M.S.; Bandoni, J.A. Supply chain optimization of large-scale continuous processes. Comput. Chem. Eng. 2005, 29, 1305-1316.

[5] Han, J.; Lee, I. Multiperiod stochastic optimization model for carbon capture and storage infrastructure under uncertainty in $\mathrm{CO}_{2}$ emissions, product prices and operating costs. Ind. Eng. Chem. Res. 2012, 51, 11445-11457.

[6] Awudu, I.; Zhang, J. Uncertainties and sustainability concepts in biofuel supply chain management: A review. Renewable and Sustainable Energy Reviews. 2012, 16, 1359-1368.

[7] Eksioglu, D.S.; Acharya, A.; Leightly, L.E.; Arora, S. Analysing the design and management of biomass-to-biorefineries supply chain. Computers and Industrial Engineering. 2009, 13421352.

[8] Kim, J.; Realff, M.J.; Lee, J.H. Optimal design and global sensitivity analysis of biomass supply chain networks for biofuels under uncertainty. Computers and Chemical Engineering. 2011, 35, 1738-1751.

[9] Susarla, N.; Karimi, I. A. Integrated supply chain planning for multinational pharmaceutical enterprises. Comput. Chem. Eng. 2012, 42, 168-177.

[10] Sundaramoorthy, A.; Evans, J. M. B.; Barton, P. I. Capacity planning under clinical trials uncertainty in continuous pharmaceutical manufacturing, 1: mathematical framework. Ind. Eng. Chem. Res. 2012, 51, 13692-13702. 
[11] Papageorgiou, L. G. Supply chain optimisation for the process industries: Advances and opportunities. Computers \& Chemical Engineering 2009, 33, 1931-1938.

[12] Nikolopoulou, A.; Ierapetritou, M. G. Optimal design of sustainable chemical processes and supply chains: A review. Computers \& Chemical Engineering 2012, 44, 94-103.

[13] Grossmann I. E.; Guillén-Gosálbez G. Scope for the application of mathematical programming techniques in the synthesis and planning of sustainable processes. Computers \& Chemical Engineering 2010, 34, 1365-1376.

[14] Sahinidis N. V. Optimization under uncertainty: state-of-the-art and opportunities. Computers \& Chemical Engineering 2004, 28, 971-983.

[15] Birge, J. R.; Louveaux, F. Introduction to stochastic programming, Second Edition; Springer, New York, 2011.

[16] Ben-Tal, A.; Ghaoui, L. E.; Nemirovski, A. Robust Optimization; Princeton University Press, NJ, 2009.

[17] McLean, K.; Li, X. Robust scenario formulations for strategic supply chain optimization under uncertainty. Ind. Eng. Chem. Res. 2013, 52, 5721-5734.

[18] Benders, J. F. Partitioning procedures for solving mixed-variables programming problems. Numerische Mathematik 1962, 4, 238-252.

[19] Van Slyke R. M.; Wets R. L-shaped linear programs with applications to optimal control and stochastic programming. SIAM J. Appl. Math. 1969, 17, 638-633.

[20] Dantzig, G. B.; Wolfe, P. Decomposition principle for linear programs. Oper. Res. 1960, 8, 
101-111.

[21] Fisher, M. L. The Lagrangian relaxation method for solving integer programming problems. Management Science 1981, 27, 1-18.

[22] Carøe, C. C.; Schultz, R. Dual decomposition in stochastic integer programming. Operations Research Letters 1999, 24, 37-45.

[23] Barnhart C., Johnson, E. L., Nemhauser G. L., Savelsbergh, M. W. P., Vance P. H. Branchand-price: Column generation for solving huge integer programs. Operations Research 1998, 46, 316-329.

[24] Bertsimas D., Pachamanova, D., Sim, M. Robust linear optimization under general norms. Operations Research Letters 2004, 32, 510-516.

[25] Rosenthal, R. E. GAMS: A User's Manual; GAMS DevelopmentCorporation: Washington, DC, 2008.

[26] Moler, C. Numerical Computing with MATLAB; The MathWorks, Inc., Natick, MA, 2004.

[27] IBM ILOG CPLEX Optimizer: High-performance mathematicalprogramming solver for linear programming, mixed integer programming , and quadratic programming. http://www01.ibm.com/software/integration/optimization/cplex-optimizer/, accessed on May 2, 2014.

[28] Li, X., Tomasgard, A., Barton, P. Nonconvex generalized Benders decomposition for stochastic separable mixed-integer nonlinear programs. Journal of Optimization Theory and Applications 2011, 151, 425-454. 


\section{Nomenclature for the Deterministic SCO Problem}

\section{Indices and Sets}

$i \in I=\{1, \ldots, 5\}-$ Plants

$j \in J=\{1, \ldots, 5\}-$ Regional warehouses

$k \in K=\{1, \ldots, 5\}-$ Regional markets

$n \in N$ - Time periods

$u \in U=\{1, \ldots, 55\}-$ PRM grades

$v \in V=\{1, \ldots, 23\}-$ FP grades

$w \in W=\{1, \ldots, 41\}-$ Impurities

$(i, j) \in \Omega-$ FP shipment routes for plants to regional warehouses

$(i, k) \in \Theta-$ FP shipment routes for plants to regional markets

$(i, v) \in \Psi-$ FP grades available for production at each plant

$(j, k) \in \Pi-\mathrm{FP}$ shipment routes for regional warehouses to regional markets

\section{Parameters}

$a_{i}^{\text {avg,FP }}-$ Average additive factor for plant $i$

$b_{i}^{\text {td }}-$ Intercept related to minimum turndown for plant $i$

$b_{i}^{\mathrm{wl}}-$ Intercept related to waste limit for plant $i$

$C^{\text {pen }}-$ Penalty cost for not meeting demand requirements, $\$ / \mathrm{T}$

$C^{\text {cap }}$ - Investment cost per unit capacity, $\$ / T$

$C_{i}^{\mathrm{fix}}-$ Fixed cost for plant $i, \$$

$C_{i}^{\mathrm{var}}-$ Other variable costs for plant $i, \$ / \mathrm{T}$

$C_{i, k}^{\mathrm{fr}, \mathrm{FP}, \mathrm{PD}}-$ Freight cost of FP from plant $i$ to market $k, \$ / \mathrm{T}$

$C_{i, j}^{\mathrm{fr}, \mathrm{FP}, \mathrm{PW}}-$ Freight cost of FP from plant $i$ to regional warehouse $j, \$ / \mathrm{T}$

$C_{j, k}^{\mathrm{fr}, \mathrm{FP}, \mathrm{WD}}-$ Freight cost of FP from regional warehouse $j$ to market $k, \$ / \mathrm{T}$

$C_{i}^{\mathrm{PI}}-$ Plant $i$ on-site inventory cost, $\$ / \mathrm{T}$

$C_{j}^{\mathrm{WI}}-$ Regional warehouse $j$ inventory cost, $\$ / \mathrm{T}$

$C_{i, u}^{\mathrm{PRM}}-$ Cost of PRM grade $u$ for plant $i, \$ / \mathrm{T}$

$C_{i}^{\mathrm{RM} 2}-$ Cost of RM2 for plant $i, \$ / \mathrm{T}$

$C_{i}^{\mathrm{RM} 3}-$ Cost of RM3 for plant $i, \$ / \mathrm{T}$ 
$C_{i}^{\text {waste }}-$ Cost of waste for plant $i, \$ / \mathrm{T}$

$D_{i, v}^{\mathrm{P}}-$ Demand of FP grade $v$ at plant $i, \mathrm{~T}$

$D_{j, v}^{W}$ - Demand of FP grade $v$ at regional warehouse $j, \mathrm{~T}$

$D_{v, k}^{\min }-$ Minimum demand of FP grade $v$ from at market $k, \mathrm{~T}$

$D_{v, k}^{\max }$ - Maximum demand of FP grade $v$ from at market $k, \mathrm{~T}$

$E_{u}^{\mathrm{PRM}}-$ Effective percentage in PRM grade $u$, for generating FP, $\%$

$m_{i}^{\mathrm{td}}-$ Slope related to minimum turndown for plant $i$

$m_{i}^{\mathrm{wl}}-$ Slope related to waste limit for plant $i$

$M_{u}^{\mathrm{PRM}}-\mathrm{PRM}$ grade $u$ availability, T

$O_{i}$ - Scheduled outage at plant $i$, day/year

$P_{k, v}^{\mathrm{FP}}-$ Price of FP grade $v$ for market $k, \$ / \mathrm{T}$

$\hat{P}_{j, v}^{\mathrm{FP}}-$ Estimated price of FP grade $v$ for regional warehouse $j, \$ / \mathrm{T}$

$q_{i, u}^{\mathrm{RM} 2}-\mathrm{RM} 2$ to PRM grade $u$ ratio for plant $i$

$q_{i, u}^{\mathrm{RM} 3}-\mathrm{RM} 3$ to PRM grade $u$ ratio for plant $i$

$q_{u}^{\text {waste }}-$ Waste to PRM grade $u$ ratio, \%

$q_{u, w}^{\text {imp }}$ - Impurity $w$ content in PRM grade $u, \%$

$q_{i, j}^{\mathrm{fr}, \mathrm{PW}}$ - Proportion of FP shipped from plant $i$ to regional warehouse $j, \%$

$Q_{i, w}^{\mathrm{imp}}-$ Maximum impurity $w$ limit for PRM at plant $i, \%$ or PPM

$Q_{i}^{\max }-$ Maximum limit for total blend at plant $i, \%$

$r_{i}^{\text {inc }}-$ Income tax rate for plant $i, \%$

$r_{i, k}^{\mathrm{du}}-$ Duty rate for shipments from plant $i$ to market $k, \%$

$\hat{r}_{i, j}^{\mathrm{du}}-$ Estimated duty rate for shipments from plant $i$ to regional warehouse $j, \%$

$r^{\text {tp }}-$ Transfer price rate, $\%$

$R_{i, v}^{\mathrm{FP}}$ - Target inventory day supply of FP grade $v$ for plant $i$, day

$R_{j, v}^{\mathrm{FP}}-$ Target inventory day supply of FP grade $v$ for regional warehouse $j$, day

$R_{i, u}^{\mathrm{PRM}, \mathrm{P}}-$ Target inventory of PRM grade $u$ for plant $i, \mathrm{~T}$

$R_{u}^{\mathrm{PRM}, \mathrm{W}}-$ Target inventory of PRM grade $u$ for the PRM warehouse, T 
$U_{i}-$ Uptime for plant $i, \%$

$X_{i, v}^{\mathrm{FP}, \mathrm{P}}-$ Beginning inventory of FP grade $v$ at plant $i, \mathrm{~T}$

$X_{j, v}^{\mathrm{FP}, \mathrm{W}}-$ Beginning inventory of FP grade $v$ at regional warehouse $j, \mathrm{~T}$

$X_{i, u}^{\mathrm{PRM}, \mathrm{P}}-$ Beginning inventory of PRM grade $u$ at plant $i, \mathrm{~T}$

$X_{u}^{\mathrm{PRM}, \mathrm{W}}-$ Beginning inventory of PRM grade $u$ at the PRM warehouse, T

$Y_{i}^{\mathrm{FP}}-$ Yield of FP at plant $i, \%$

$Z_{i}^{\max }-$ Maximum allowable capacity at plant $i, \mathrm{~T}$

\section{Variables}

$c_{i}^{\mathrm{fr}}-$ Freight cost for plant $i, \$$

$c_{i}^{\mathrm{du}}-$ Duty cost for plant $i, \$$

$c_{i}^{\mathrm{I}}-$ Inventory cost for plant $i, \$$

$c_{i}^{\text {PRM }}-$ PRM cost for plant $i, \$$

$c_{i}^{\mathrm{RM} 2}-\mathrm{RM} 2$ cost for plant $i, \$$

$c_{i}^{\mathrm{RM} 3}-\mathrm{RM} 3$ cost for plant $i, \$$

$c_{i}^{\text {waste }}-$ Waste cost for plant $i, \$$

$c_{i}^{\mathrm{OPVC}}-$ Other plant $i$ variable costs, $\$$

$c_{i}^{\text {cap }}$ - Capacity cost for plant $i, \$$

$c_{v, k}^{\text {pen }}-$ Penalty cost of FP grade $v$ at market $k, \$$

$f_{i, k, v}^{\mathrm{FP}, \mathrm{PD}}-$ Shipment of FP grade $v$ from plant $i$ to market $k, \mathrm{~T}$

$f_{i, j, v}^{\mathrm{FP}, \mathrm{PW}}-$ Shipment of FP grade $v$ from plant $i$ to regional warehouse $j, \mathrm{~T}$

$f_{j, k, v}^{\mathrm{FP}, \mathrm{WD}}-$ Shipment of FP grade $v$ from regional warehouse $j$ to market $k, \mathrm{~T}$

$f_{i, u}^{\text {PRM,c }}-$ PRM grade $u$ consumed at plant $i, \mathrm{~T}$

$f_{i, u}^{\mathrm{PRM}, \mathrm{P}}-\mathrm{PRM}$ grade $u$ purchased and sent to plant $i, \mathrm{~T}$

$f_{u}^{\text {PRM,W }}-$ PRM grade $u$ purchased and sent to the PRM warehouse, T

$f_{i, u}^{\mathrm{PRM}, \mathrm{WP}}-$ Shipments of PRM grade $u$ from the PRM warehouse to plant $i, \mathrm{~T}$

$f_{i, v}^{\mathrm{FP}, \mathrm{p}}-$ Amount of FP grade $v$ produced from plant $i, \mathrm{~T}$

$z_{i}$ - Capacity of plant $i, \mathrm{~T}$ 


\section{Appendix A: Transformation of Uncertain Linear Equality Constraints for Formulation (AARC_IN)}

Consider the following linear equality constraint,

$$
(\alpha \xi+\beta)^{T} x+w^{T} y=0
$$

where $x \in \mathbb{R}^{n_{x}}$ and $y \in \mathbb{R}^{n_{y}}$ denote first and second stage variables, respectively, $\xi \in \mathbb{R}^{n_{\xi}}$ denotes uncertain parameters, and $\alpha, \beta, w$ denote deterministic parameters with conformable dimensions. Assume, without loss of generality, that none of the component in $\xi$ is linearly dependent on the other components. Eq. (A.1) can be expressed as the following two inequalities:

$$
\begin{gathered}
(\alpha \xi+\beta)^{T} x+w^{T} y \leq 0 \\
(-\alpha \xi-\beta)^{T} x-w^{T} y \leq 0
\end{gathered}
$$

For the robust scenario formulation (AARS_IN), the uncertainty region is partitioned into $s$ subregions $\Xi_{\omega}=\left\{\xi_{\omega}:\left\|M_{\omega}\left(\xi_{\omega}-\bar{\xi}_{\omega}\right)\right\|_{\infty} \leq \delta_{\omega}\right\}(\omega=1, \cdots, s)$, and the second-stage variables $y$ are represented by affine functions $U_{\omega} \xi_{\omega}+v_{\omega}$ for each uncertainty subregion. Then according to Subsection 3.1, Eq. (A.2) can be transformed into the following $s$ groups of deterministic inequality constraints:

$$
\beta^{T} x+w^{T} v_{\omega}+\left(x^{T} \alpha+w^{T} U_{\omega}\right) \bar{\xi}_{\omega}+\delta_{\omega}\left\|\left(x^{T} \alpha+w_{i}^{T} U_{\omega}\right) M_{\omega}^{-1}\right\|_{1} \leq 0, \quad \omega=1, \ldots, s
$$

Similarly, Eq. (A.3) can be transformed into

$$
-\beta^{T} x-w^{T} v_{\omega}-\left(x^{T} \alpha+w^{T} U_{\omega}\right) \bar{\xi}_{\omega}+\delta_{\omega}\left\|\left(x^{T} \alpha+w^{T} U_{\omega}\right) M_{\omega}^{-1}\right\|_{1} \leq 0, \quad \omega=1, \ldots, s
$$

$(\mathrm{A} .4)+(\mathrm{A} .5)$ gives

$$
\delta_{\omega}\left\|\left(x^{T} \alpha+w^{T} U_{\omega}\right) M_{\omega}^{-1}\right\|_{1} \leq 0, \quad \omega=1, \ldots, s
$$


or

$$
x^{T} \alpha+w^{T} U_{\omega}=0, \quad \omega=1, \ldots, s .
$$

With Eq. (A.6), Eqs. (A.4) and (A.5) become,

$$
\begin{array}{rr}
\beta^{T} x+w^{T} v_{\omega} \leq 0, & \omega=1, \ldots, s, \\
-\beta^{T} x-w^{T} v_{\omega} \leq 0, & \omega=1, \ldots, s,
\end{array}
$$

so

$$
\beta^{T} x+w^{T} v_{\omega}=0, \quad \omega=1, \ldots, s .
$$

Therefore, the uncertain linear inequality (A.1) is expressed as two groups of deterministic constraints (A.7) and (A.10).

\section{Appendix B: The Robust Scenario Formulation for the SCO Problem With the Demand Uncertainties}

The formulation is given below:

$$
\max \sum_{\omega=1}^{s} p_{\omega}\left[\sum_{i \in I}\left(\text { Revenue }_{i, \omega}-\mathrm{TC}_{i, \omega}\right)\left(1-r_{i}^{\mathrm{inc}}\right)\right]
$$

s.t. Revenue $_{i, \omega}=\sum_{(i, k) \in \Theta} \sum_{v \in V}\left(\left(\Phi_{i, k, v, \omega}^{\mathrm{FP}, \mathrm{PD}} \cdot \bar{\xi}_{\omega}+\phi_{i, k, v, \omega}^{\mathrm{FP}, \mathrm{PD}}\right) \cdot P_{k, v}^{\mathrm{FP}}\right)+\sum_{(i, j) \in \Omega} \sum_{v \in V}\left(\left(\Phi_{i, j, v, \omega}^{\mathrm{FP}, \mathrm{PW}} \cdot \bar{\xi}_{\omega}+\phi_{i, j, v, \omega}^{\mathrm{FP}, \mathrm{PW}}\right) \cdot \hat{P}_{j, v}^{\mathrm{FP}}\right)$,

$$
i \in I, \quad \omega=1, \cdots, s,
$$

$$
\begin{array}{r}
\mathrm{TC}_{i, \omega}=c_{i}^{\mathrm{fix}}+c_{i}^{\mathrm{cap}}+\bar{c}_{i, \omega}^{\mathrm{fr}}+\bar{c}_{i, \omega}^{\mathrm{du}}+\bar{c}_{i, \omega}^{\mathrm{I}}+\bar{c}_{i, \omega}^{\mathrm{PRM}}+\bar{c}_{i, \omega}^{\mathrm{RM} 2}+\bar{c}_{i, \omega}^{\mathrm{RM} 3}+\bar{c}_{i, \omega}^{\mathrm{waste}}+\bar{c}_{i, \omega}^{\mathrm{OPVC}}, \\
\quad i \in I, \omega=1, \cdots, s,
\end{array}
$$




$$
\begin{aligned}
c_{i}^{\mathrm{cap}} & =z_{i} \cdot C^{\mathrm{cap}}, \\
i \in I, & \\
\bar{c}_{i, \omega}^{\mathrm{fr}} & =\sum_{(i, k) \in \Theta} \sum_{v \in V}\left(\left(\Phi_{i, k, v, \omega}^{\mathrm{FP}, \mathrm{PD}} \cdot \bar{\xi}_{\omega}+\phi_{i, k, v, \omega}^{\mathrm{FP}, \mathrm{PD}}\right) \cdot C_{i, k}^{\mathrm{fr}, \mathrm{FP}, \mathrm{PD}}\right)+\sum_{(i, j) \in \Omega} \sum_{v \in V}\left(\left(\Phi_{i, j, v, \omega}^{\mathrm{FP}, \mathrm{PW}} \cdot \bar{\xi}_{\omega}+\phi_{i, j, v, \omega}^{\mathrm{FP}, \mathrm{PW}}\right) \cdot C_{i, j}^{\mathrm{fr}, \mathrm{FP}, \mathrm{PW}}\right) \\
& +\sum_{(i, j) \in \Omega} \sum_{(j, k) \in \Pi} \sum_{v \in V}\left(\left(\Phi_{j, k, v, \omega}^{\mathrm{FP}, \mathrm{WD}} \cdot \bar{\xi}_{\omega}+\phi_{j, k, v, \omega}^{\mathrm{FP}, \mathrm{WD}}\right) \cdot C_{j, k}^{\mathrm{fr}, \mathrm{FP}, \mathrm{WD}} \cdot q_{i, j}^{\mathrm{fr}, \mathrm{PW}}\right),
\end{aligned}
$$

$$
i \in I, \quad \omega=1, \cdots, s,
$$

$$
\begin{aligned}
\bar{c}_{i, \omega}^{\mathrm{du}}= & \sum_{(i, k) \in \Theta} \sum_{v \in V}\left(\left(\Phi_{i, k, v, \omega}^{\mathrm{FP}, \mathrm{PD}} \cdot \bar{\xi}_{\omega}+\phi_{i, k, v, \omega}^{\mathrm{FP}, \mathrm{PD}}\right) \cdot P_{k, v}^{\mathrm{FP}} \cdot r_{i, k}^{\mathrm{du}}\right) \cdot r^{\mathrm{tp}} \\
& +\sum_{(i, j) \in \Omega} \sum_{v \in V}\left(\left(\Phi_{i, j, v, \omega}^{\mathrm{FP}, \mathrm{PW}} \cdot \bar{\xi}_{\omega}+\phi_{i, j, v, \omega}^{\mathrm{FP}, \mathrm{PW}}\right) \cdot \hat{P}_{j, v}^{\mathrm{FP}} \cdot \hat{r}_{i, j}^{\mathrm{du}}\right) \cdot r^{\mathrm{tp}},
\end{aligned}
$$

$$
i \in I, \quad \omega=1, \cdots, s,
$$

$\bar{c}_{i, \omega}^{\mathrm{I}}=\sum_{(i, v) \in \Psi}\left(\left(\Phi_{i, v, \omega}^{\mathrm{FP}, \mathrm{p}} \cdot \bar{\xi}_{\omega}+\phi_{i, v, \omega}^{\mathrm{FP}, \mathrm{p}}\right) \cdot C_{i}^{\mathrm{PI}}\right)+\sum_{(i, j) \in \Omega} \sum_{v \in V}\left(\left(\Phi_{i, j, v, \omega}^{\mathrm{FP}, \mathrm{PW}} \cdot \bar{\xi}_{\omega}+\phi_{i, j, v, \omega}^{\mathrm{FP}, \mathrm{PW}}\right) \cdot C_{j}^{\mathrm{WI}}\right)$,

$$
i \in I, \quad \omega=1, \cdots, s,
$$

$$
\bar{c}_{i, \omega}^{\mathrm{PRM}}=\sum_{u \in U}\left(\Phi_{i, u, \omega}^{\mathrm{PRM}, \mathrm{c}} \cdot \bar{\xi}_{\omega}+\phi_{i, u, \omega}^{\mathrm{PRM}, \mathrm{c}}\right) \cdot C_{i, u}^{\mathrm{PRM}}, \quad i \in I, \quad \omega=1, \cdots, s,
$$

$$
\bar{c}_{i, \omega}^{\mathrm{RM} 2}=\sum_{u \in U}\left(\Phi_{i, u, \omega}^{\mathrm{PRM}, \mathrm{c}} \cdot \bar{\xi}_{\omega}+\phi_{i, u, \omega}^{\mathrm{PRM}, \mathrm{c}}\right) \cdot q_{i, u}^{\mathrm{RM} 2} \cdot C_{i}^{\mathrm{RM} 2} \quad i \in I, \quad \omega=1, \cdots, s,
$$$$
\bar{c}_{i, \omega}^{\mathrm{RM} 3}=\sum_{u \in U}\left(\Phi_{i, u, \omega}^{\mathrm{PRM}, \mathrm{c}} \cdot \bar{\xi}_{\omega}+\phi_{i, u, \omega}^{\mathrm{PRM}, \mathrm{c}}\right) \cdot q_{i, u}^{\mathrm{RM} 3} \cdot C_{i}^{\mathrm{RM} 3} \quad i \in I, \quad \omega=1, \cdots, s,
$$$$
\bar{c}_{i, \omega}^{\text {waste }}=\sum_{u \in U}\left(\Phi_{i, u, \omega}^{\mathrm{PRM}, \mathrm{c}} \cdot \bar{\xi}_{\omega}+\phi_{i, u, \omega}^{\mathrm{PRM}, \mathrm{c}}\right) \cdot q_{i, u}^{\text {waste }} \cdot C_{i}^{\text {waste }} \quad i \in I, \omega=1, \cdots, s,
$$

$$
\bar{c}_{i, \omega}^{\mathrm{OPVC}}=\sum_{(i, v) \in \Psi}\left(\Phi_{i, v, \omega}^{\mathrm{FP}, \mathrm{p}} \cdot \bar{\xi}_{\omega}+\phi_{i, v, \omega}^{\mathrm{FP}, \mathrm{p}}\right) \cdot C_{i}^{\mathrm{var}} \quad i \in I, \quad \omega=1, \cdots, s,
$$




$$
\begin{aligned}
& \left(\sum_{u \in U} \Phi_{i, u}^{\mathrm{PRM}, \mathrm{c}}-\sum_{(i, v) \in \Psi} \eta_{i}^{t d, 1} \Phi_{i, v}^{\mathrm{FP}, \mathrm{p}}\right) \bar{\xi}_{\omega}+\delta_{\omega}\left\|\left(\sum_{u \in U} \Phi_{i, u}^{\mathrm{PRM}, \mathrm{c}}-\sum_{(i, v) \in \Psi} \eta_{i}^{t d, 1} \Phi_{i, v}^{\mathrm{FP}, \mathrm{p}}\right) M^{-1}\right\|_{1} \\
& +\left(\sum_{u \in U} \phi_{i, u}^{\mathrm{PRM}, \mathrm{c}}-\sum_{(i, v) \in \Psi} \eta_{i}^{t d, 1} \phi_{i, v}^{\mathrm{FP}, \mathrm{p}}\right) \leq \eta_{i}^{t d, 2}
\end{aligned}
$$

$$
i \in I, \quad \omega=1, \cdots, s,
$$

$$
\begin{aligned}
& \left(\sum_{u \in U} \Phi_{i, u}^{\mathrm{PRM}, \mathrm{c}}-\sum_{(i, v) \in \Psi} \eta_{i}^{w l, 1} \Phi_{i, v}^{\mathrm{FP}, \mathrm{p}}\right) \bar{\xi}_{\omega}+\delta_{\omega}\left\|\left(\sum_{u \in U} \Phi_{i, u}^{\mathrm{PRM}, \mathrm{c}}-\sum_{(i, v) \in \Psi} \eta_{i}^{w l, 1} \Phi_{i, v}^{\mathrm{FP}, \mathrm{p}}\right) M^{-1}\right\|_{1} \\
& +\left(\sum_{u \in U} \phi_{i, u}^{\mathrm{PRM}, \mathrm{c}}-\sum_{(i, v) \in \Psi} \eta_{i}^{w l, 1} \phi_{i, v}^{\mathrm{FP}, \mathrm{p}}\right) \leq \eta_{i}^{w l, 2}
\end{aligned}
$$

$$
i \in I, \quad \omega=1, \cdots, s,
$$

$$
\left(\sum_{(i, v) \in \Psi} \Phi_{i, v, \omega}^{\mathrm{FP}, p}\right) \bar{\xi}_{\omega}+\delta_{\omega}\left\|\left(\sum_{(i, v) \in \Psi} \Phi_{i, v, \omega}^{\mathrm{FP}, p}\right) M^{-1}\right\|_{1}+\sum_{(i, v) \in \Psi} \phi_{i, v, \omega}^{\mathrm{FP}, p} \leq z_{i}, \quad i \in I, \quad \omega=1, \cdots, s,
$$

$$
z_{i} \leq Z_{i}^{\max }
$$

$i \in I$,

$$
\sum_{u \in U}\left(\Phi_{i, u, \omega}^{\mathrm{PRM}, \mathrm{c}} \cdot E_{u}^{\mathrm{PRM}}\right) \cdot a_{i}^{\mathrm{avg}, \mathrm{FP}} \cdot Y_{i}^{\mathrm{FP}}=\sum_{(i, v) \in \Psi}\left(\Phi_{i, v, \omega}^{\mathrm{FP}, \mathrm{p}}\right), \quad i \in I, \quad \omega=1, \cdots, s,
$$

$$
\begin{gathered}
\sum_{u \in U}\left(\phi_{i, u, \omega}^{\mathrm{PRM}, \mathrm{c}} \cdot E_{u}^{\mathrm{PRM}}\right) \cdot a_{i}^{\mathrm{avg}, \mathrm{FP}} \cdot Y_{i}^{\mathrm{FP}}=\sum_{(i, v) \in \Psi}\left(\phi_{i, v, \omega}^{\mathrm{FP}, \mathrm{p}}\right), \\
\left(\sum_{i \in I} \Phi_{i, u, \omega}^{\mathrm{PRM}, \mathrm{P}}+\Phi_{u, \omega}^{\mathrm{PRM}, \mathrm{W}}\right) \bar{\xi}_{\omega}+\delta_{\omega}\left\|\left(\sum_{i \in I} \Phi_{i, u, \omega}^{\mathrm{PRM}, \mathrm{P}}+\Phi_{u, \omega}^{\mathrm{PRM}, \mathrm{W}}\right) M^{-1}\right\|_{1}+\sum_{i \in I} \phi_{i, u, \omega}^{\mathrm{PRM}, \mathrm{P}}+\phi_{u, \omega}^{\mathrm{PRM}, \mathrm{W}} \leq M_{u}^{\mathrm{PRM}},
\end{gathered}
$$

$$
u \in U, \omega=1, \cdots, s,
$$




$$
\begin{aligned}
& \left(\Phi_{u, \omega}^{\mathrm{PRM}, \mathrm{W}}-\sum_{i \in I} \Phi_{i, u, \omega}^{\mathrm{PRM}, \mathrm{WP}}\right) \bar{\xi}_{\omega}-\delta_{\omega}\left\|\left(\Phi_{u, \omega}^{\mathrm{PRM}, \mathrm{W}}-\sum_{i \in I} \Phi_{i, u, \omega}^{\mathrm{PRM}, \mathrm{WP}}\right) M^{-1}\right\|_{1} \\
& +\phi_{u, \omega}^{\mathrm{PRM}, \mathrm{W}}-\sum_{i \in I} \phi_{i, u, \omega}^{\mathrm{PRM}, \mathrm{WP}} \geq R_{u}^{\mathrm{PRM}, \mathrm{W}}-X_{u}^{\mathrm{PRM}, \mathrm{W}}, \\
& u \in U, \omega=1, \cdots, s, \\
& \left(\Phi_{i, u, \omega}^{\mathrm{PRM}, \mathrm{P}}+\Phi_{i, u, \omega}^{\mathrm{PRM}, \mathrm{WP}}-\Phi_{i, u, \omega}^{\mathrm{PRM}, \mathrm{c}}\right) \bar{\xi}_{\omega}-\delta_{\omega}\left\|\left(\Phi_{i, u, \omega}^{\mathrm{PRM}, \mathrm{P}}+\Phi_{i, u, \omega}^{\mathrm{PRM}, \mathrm{WP}}-\Phi_{i, u, \omega}^{\mathrm{PRM}, \mathrm{c}}\right) M^{-1}\right\|_{1} \\
& +\left(\phi_{i, u, \omega}^{\mathrm{PRM}, \mathrm{P}}+\phi_{i, u, \omega}^{\mathrm{PRM}, \mathrm{WP}}-\phi_{i, u, \omega}^{\mathrm{PRM}, \mathrm{c}}\right) \geq R_{i, u}^{\mathrm{PRM}, \mathrm{P}}-X_{i, u}^{\mathrm{PRM}, \mathrm{P}} \\
& i \in I, \quad u \in U, \quad \omega=1, \cdots, s, \\
& \left(\Phi_{i, v, \omega}^{\mathrm{FP}, \mathrm{p}}-\sum_{(i, j) \in \Omega} \Phi_{i, j, v, \omega}^{\mathrm{FP}, \mathrm{PW}}-\sum_{(i, k) \in \Theta} \Phi_{i, k, v, \omega}^{\mathrm{FP}, \mathrm{PD}}\right) \bar{\xi}_{\omega}+\phi_{i, v, \omega}^{\mathrm{FP}, \mathrm{p}}-\sum_{(i, j) \in \Omega} \phi_{i, j, v, \omega}^{\mathrm{FP}, \mathrm{PW}}-\sum_{(i, k) \in \Theta} \phi_{i, k, v, \omega}^{\mathrm{FP}, \mathrm{PD}} \\
& -\delta_{\omega}\left\|\left(\Phi_{i, v, \omega}^{\mathrm{FP}, \mathrm{p}}-\sum_{(i, j) \in \Omega} \Phi_{i, j, v, \omega}^{\mathrm{FP}, \mathrm{PW}}-\sum_{(i, k) \in \Theta} \Phi_{i, k, v, \omega}^{\mathrm{FP}, \mathrm{PD}}\right) M^{-1}\right\|_{1} \geq D_{i, v}^{\mathrm{P}} \cdot R_{i, v}^{\mathrm{FP}} / 365-X_{i, v}^{\mathrm{FP}, \mathrm{P}}, \\
& (i, v) \in \Psi, \omega=1, \cdots, s \\
& \left(\sum_{i \in I} \Phi_{i, j, v, \omega}^{\mathrm{FP}, \mathrm{PW}}-\sum_{(j, k) \in \Pi} \Phi_{j, k, v, \omega}^{\mathrm{FP}, \mathrm{WD}}\right) \bar{\xi}_{\omega}+\sum_{i \in I} \phi_{i, j, v, \omega}^{\mathrm{FP}, \mathrm{PW}}-\sum_{(j, k) \in \Pi} \phi_{j, k, v, \omega}^{\mathrm{FP}, \mathrm{WD}} \\
& -\delta_{\omega}\left\|\left(\sum_{i \in I} \Phi_{i, j, v, \omega}^{\mathrm{FP}, \mathrm{PW}}-\sum_{(j, k) \in \Pi} \Phi_{j, k, v, \omega}^{\mathrm{FP}, \mathrm{WD}}\right) M^{-1}\right\|_{1} \geq D_{j, v}^{W} \cdot R_{j, v}^{\mathrm{FP}} / 365-X_{j, v}^{\mathrm{FP}, \mathrm{W}}, \\
& j \in J, v \in V, \omega=1, \cdots, s
\end{aligned}
$$




$$
\begin{aligned}
& \sum_{(i, k) \in \Theta} \phi_{i, k, v, \omega}^{\mathrm{FP}, \mathrm{PD}}+\sum_{(j, k) \in \Pi} \phi_{j, k, v, \omega}^{\mathrm{FP}, \mathrm{WD}}+\left(\sum_{(i, k) \in \Theta} \Phi_{i, k, v, \omega}^{\mathrm{FP}, \mathrm{PD}}+\sum_{(j, k) \in \Pi} \Phi_{j, k, v, \omega}^{\mathrm{FP}, \mathrm{WD}}-\bar{D}_{v, k}^{\mathrm{min}} \gamma_{k}\right) \bar{\xi}_{\omega} \\
& -\delta_{\omega}\left\|\left(\sum_{(i, k) \in \Theta} \Phi_{i, k, v, \omega}^{\mathrm{FP}, \mathrm{PD}}+\sum_{(j, k) \in \Pi} \Phi_{j, k, v, \omega}^{\mathrm{FP}, \mathrm{WD}}-\bar{D}_{v, k}^{\mathrm{min}} \gamma_{k}\right) M^{-1}\right\|_{1} \geq 0,
\end{aligned}
$$

$$
\begin{aligned}
& {\left[\sum_{u \in U}\left(\Phi_{i, u, \omega}^{\mathrm{PRM}, \mathrm{c}} \cdot q_{u, w}^{\mathrm{imp}}\right)-\sum_{u \in U}\left(\Phi_{i, u, \omega}^{\mathrm{PRM}, \mathrm{c}} \cdot Q_{i, w}^{\mathrm{imp}}\right)\right] \bar{\xi}_{\omega}+\sum_{u \in U}\left(\phi_{i, u, \omega}^{\mathrm{PRM}, \mathrm{c}} \cdot q_{u, w}^{\mathrm{imp}}\right)-\sum_{u \in U}\left(\phi_{i, u, \omega}^{\mathrm{PRM}, \mathrm{c}} \cdot Q_{i, w}^{\mathrm{imp}}\right)} \\
& +\delta_{\omega}\left\|\left[\sum_{u \in U}\left(\Phi_{i, u, \omega}^{\mathrm{PRM}, \mathrm{c}} \cdot q_{u, w}^{\mathrm{imp}}\right)-\sum_{u \in U}\left(\Phi_{i, u, \omega}^{\mathrm{PRM}, \mathrm{c}} \cdot Q_{i, w}^{\mathrm{imp}}\right)\right] M^{-1}\right\|_{1} \leq 0,
\end{aligned}
$$

$$
\begin{aligned}
& {\left[\sum_{u \in U}\left(\Phi_{i, u, \omega}^{\mathrm{PRM}, \mathrm{c}} \cdot E_{u}^{\mathrm{PRM}}\right)-\sum_{u \in U}\left(\Phi_{i, u, \omega}^{\mathrm{PRM}, \mathrm{c}} \cdot Q_{i}^{\mathrm{imp}}\right)\right] \bar{\xi}_{\omega}+\sum_{u \in U}\left(\phi_{i, u, \omega}^{\mathrm{PRM}, \mathrm{c}} \cdot E_{u}^{\mathrm{PRM}}\right)-\sum_{u \in U}\left(\phi_{i, u, \omega}^{\mathrm{PRM}, \mathrm{c}} \cdot Q_{i}^{\mathrm{imp}}\right)} \\
& +\delta_{\omega}\left\|\left[\sum_{u \in U}\left(\Phi_{i, u, \omega}^{\mathrm{PRM}, \mathrm{c}} \cdot E_{u}^{\mathrm{PRM}}\right)-\sum_{u \in U}\left(\Phi_{i, u, \omega}^{\mathrm{PRM}, \mathrm{c}} \cdot Q_{i}^{\mathrm{imp}}\right)\right] M^{-1}\right\|_{1} \leq 0,
\end{aligned}
$$

$$
i \in I, \omega=1, \cdots, s,
$$

$$
\Phi_{i, k, v, \omega}^{\mathrm{FP}, \mathrm{PD}} \bar{\xi}_{\omega}+\phi_{i, k, v, \omega}^{\mathrm{FP}, \mathrm{PD}}-\delta_{\omega}\left\|\Phi_{i, k, v, \omega}^{\mathrm{FP}, \mathrm{PD}} M^{-1}\right\|_{1} \geq 0, \quad i \in I, k \in K, v \in V, \omega=1, \cdots, s
$$

$$
\Phi_{i, j, v, \omega}^{\mathrm{FP}, \mathrm{PW}} \bar{\xi}_{\omega}+\phi_{i, j, v, \omega}^{\mathrm{FP}, \mathrm{PW}}-\delta_{\omega}\left\|\Phi_{i, j, v, \omega}^{\mathrm{FP}, \mathrm{PW}} M^{-1}\right\|_{1} \geq 0, \quad i \in I, j \in J, v \in V, \omega=1, \cdots, s
$$$$
\Phi_{j, k, v, \omega}^{\mathrm{FP}, \mathrm{WD}} \bar{\xi}_{\omega}+\phi_{j, k, v, \omega}^{\mathrm{FP}, \mathrm{WD}}-\delta_{\omega}\left\|\Phi_{j, k, v, \omega}^{\mathrm{FP}, \mathrm{WD}} M^{-1}\right\|_{1} \geq 0, \quad j \in J, k \in K, v \in V, \omega=1, \cdots, s,
$$$$
\Phi_{i, v, \omega}^{\mathrm{FP}, \mathrm{p}} \bar{\xi}_{\omega}+\phi_{i, v, \omega}^{\mathrm{FP}, \mathrm{p}}-\delta_{\omega}\left\|\Phi_{i, v, \omega}^{\mathrm{FP}, \mathrm{p}} M^{-1}\right\|_{1} \geq 0, \quad i \in I, v \in V, \omega=1, \cdots, s
$$$$
\Phi_{i, u, \omega}^{\mathrm{PRM}, \mathrm{c}} \bar{\xi}_{\omega}+\phi_{i, u, \omega}^{\mathrm{PRM}, \mathrm{c}}-\delta_{\omega}\left\|\Phi_{i, u, \omega}^{\mathrm{PRM}, \mathrm{c}} M^{-1}\right\|_{1} \geq 0, \quad i \in I, u \in U, \omega=1, \cdots, s
$$ 


$$
\begin{array}{ll}
\Phi_{i, u, \omega}^{\mathrm{PRM}, \mathrm{p}} \bar{\xi}_{\omega}+\phi_{i, u, \omega}^{\mathrm{PRM}, \mathrm{p}}-\delta_{\omega}\left\|\Phi_{i, u, \omega}^{\mathrm{PRM}, \mathrm{p}} M^{-1}\right\|_{1} \geq 0, & i \in I, u \in U, \omega=1, \cdots, s, \\
\Phi_{u, \omega}^{\mathrm{PRM}, \mathrm{W}} \bar{\xi}_{\omega}+\phi_{u, \omega}^{\mathrm{PRM}, \mathrm{W}}-\delta_{\omega}\left\|\Phi_{u, \omega}^{\mathrm{PRM}, \mathrm{W}} M^{-1}\right\|_{1} \geq 0, & u \in U, \omega=1, \cdots, s, \\
\Phi_{i, u, \omega}^{\mathrm{PRM}, \mathrm{WP}} \bar{\xi}_{\omega}+\phi_{i, u, \omega}^{\mathrm{PRM}, \mathrm{WP}}-\delta_{\omega}\left\|\Phi_{i, u, \omega}^{\mathrm{PRM}, \mathrm{WP}} M^{-1}\right\|_{1} \geq 0, & i \in I, u \in U, \omega=1, \cdots, s .
\end{array}
$$

Here Eqs. (B.1-16) are obtained from Eqs. (1-16) in the deterministic formulation (DSCO), through replacing variables for the material/product flow rates in scenario $\omega$ with affine functions of uncertainty realizations $\xi_{\omega}$. Specifically,

$$
\begin{gathered}
f_{i, k, v}^{\mathrm{FP}, \mathrm{PD}} \Rightarrow \Phi_{i, k, v, \omega}^{\mathrm{FP}, \mathrm{PD}} \xi_{\omega}+\phi_{i, k, v, \omega}^{\mathrm{FP}, \mathrm{PD}}, f_{i, j, v}^{\mathrm{FP}, \mathrm{PW}} \Rightarrow \Phi_{i, j, v, \omega}^{\mathrm{FP}, \mathrm{PW}} \xi_{\omega}+\phi_{i, j, v, \omega}^{\mathrm{FP}, \mathrm{PW}}, \\
f_{j, k, v}^{\mathrm{FP}, \mathrm{WD}} \Rightarrow \Phi_{j, k, v, \omega}^{\mathrm{FP}, \mathrm{WD}} \xi_{\omega}+\phi_{j, k, v, \omega}^{\mathrm{FP}, \mathrm{WD}}, f_{i, v}^{\mathrm{FP}, \mathrm{p}} \Rightarrow \Phi_{i, v, \omega}^{\mathrm{FP}, \mathrm{p}} \xi_{\omega}+\phi_{i, v, \omega}^{\mathrm{FP}, \mathrm{p}}, \\
f_{i, u}^{\mathrm{PRM}, \mathrm{c}} \Rightarrow \Phi_{i, u, \omega}^{\mathrm{PRM}, c} \xi_{\omega}+\phi_{i, u, \omega}^{\mathrm{PRM}, \mathrm{c}}, f_{i, u}^{\mathrm{PRM}, \mathrm{p}} \Rightarrow \Phi_{i, u, \omega}^{\mathrm{PRM}, \mathrm{p}} \xi_{\omega}+\phi_{i, u, \omega}^{\mathrm{PRM}, \mathrm{p}}, \\
f_{u}^{\mathrm{PRM}, \mathrm{W}} \Rightarrow \Phi_{u, \omega}^{\mathrm{PRM}, \mathrm{W}} \xi_{\omega}+\phi_{u, \omega}^{\mathrm{PRM}, \mathrm{W}}, f_{i, u}^{\mathrm{PRM}, \mathrm{WP}} \Rightarrow \Phi_{i, u, \omega}^{\mathrm{PRM}, \mathrm{WP}} \xi_{\omega}+\phi_{i, u, \omega}^{\mathrm{PRM}, \mathrm{WP}} .
\end{gathered}
$$

Similarly, Eqs. (B.17-18) are obtained from Eq. (17), Eqs. (B.19-26) from Eq. (18-25), and Eqs. (B.27-32) from nonnegativity of the flow rate decisions. 


\section{Appendix C: An Illustrative Example for the Construction of Robust Scenario Formulation}

Consider the following optimization problem:

$$
\begin{array}{ll}
\min & 15 x_{1}+23 x_{2}-17 z_{1}-15 z_{2} \\
\text { s.t. } & x_{1}+x_{2} \leq 20 \\
& 2.5 x_{1}-z_{1} \geq 20 \xi \\
& 0.3 x_{2}-z_{2} \geq 24
\end{array}
$$

where $x_{1}, x_{2}$ are first-stage decision variables, $z_{1}, z_{2}$ are second-stage variables, $\xi$ is an uncertain parameter that will be realized after the first-stage decisions are implemented (but before the second stage decisions are implemented). Assume that $\xi$ is uniformly distributed in $[0.4,1.6]$, then we can express the uncertainty set using the infinity-norm as $\Xi=\left\{\xi:\|M(\xi-\bar{\xi})\|_{\infty} \leq 1\right\}$, where $\bar{\xi}=1, M=5 / 3$. We can partition the uncertainty set evenly into three subsets, each can be expressed as

$$
\Xi_{\omega}=\left\{\xi_{\omega}:\left\|M\left(\xi_{\omega}-\bar{\xi}_{\omega}\right)\right\|_{\infty} \leq \delta_{\omega}\right\}, \quad \omega=1,2,3
$$

where $\omega$ indexes the three subsets, $\bar{\xi}_{1}=0.6, \bar{\xi}_{2}=1, \bar{\xi}_{3}=1.4, \delta_{1}=\delta_{2}=\delta_{3}=1 / 3$. The robust scenario formulation with the 3 scenarios can be initially written in the following form:

$$
\begin{array}{ll}
\min & 15 x_{1}+23 x_{2}+\sum_{\omega=1}^{3}\left(-17 \bar{z}_{1, \omega}-15 \bar{z}_{2, \omega}\right) \\
\text { s.t. } \quad & x_{1}+x_{2} \leq 20, \\
& 2.5 x_{1}-z_{1, \omega} \geq 20 \xi_{\omega}, \quad \forall \xi_{\omega} \in \Xi_{\omega}, \quad \omega=1,2,3, \\
& 0.3 x_{2}-z_{2, \omega} \geq 24, \quad \omega=1,2,3,
\end{array}
$$

where $\bar{z}_{1, \omega}, \bar{z}_{2, \omega}$ denote the expected values of the second-stage variables in scenario $\omega$. 
The next step is to express the second-stage variables as affine functions of $\xi_{\omega}$. Specifically, for $\omega=1,2,3$ let

$$
\begin{aligned}
& z_{1, \omega}=\alpha_{1, \omega} \xi_{\omega}+\beta_{1, \omega}, \\
& z_{2, \omega}=\alpha_{2, \omega} \xi_{\omega}+\beta_{2, \omega},
\end{aligned}
$$

where $\alpha_{1, \omega}, \beta_{1, \omega}, \alpha_{2, \omega}, \beta_{2, \omega}$ are the new second-stage decision variables. Substitute the affine functions in formulation (C.2), then we get

$$
\begin{array}{ll}
\min & 15 x_{1}+23 x_{2}+\sum_{\omega=1}^{3}\left[\left(-17 \alpha_{1, \omega}-15 \alpha_{2, \omega}\right) \bar{\xi}_{\omega}-17 \beta_{1, \omega}-15 \beta_{2, \omega}\right] \\
\text { s.t. } & x_{1}+x_{2} \leq 20, \\
& 2.5 x_{1}-\beta_{1, \omega}-\left(\alpha_{1, \omega}+20\right) \xi_{\omega} \geq 0, \quad \forall \xi_{\omega} \in \Xi_{\omega}, \quad \omega=1,2,3, \\
& 0.3 x_{2}-24-\beta_{2, \omega}-\alpha_{2, \omega} \xi_{\omega} \geq 0, \quad \forall \xi_{\omega} \in \Xi_{\omega}, \quad \omega=1,2,3,
\end{array}
$$

The last two groups of constraints in formulation (C.3) can be transformed into deterministic constraints as explained in Section 3.1, then the formulation becomes

$$
\begin{array}{ll}
\min & 15 x_{1}+23 x_{2}+\sum_{\omega=1}^{3}\left[\left(-17 \alpha_{1, \omega}-15 \alpha_{2, \omega}\right) \bar{\xi}_{\omega}-17 \beta_{1, \omega}-15 \beta_{2, \omega}\right] \\
\text { s.t. } & x_{1}+x_{2} \leq 20, \\
& 2.5 x_{1}-\beta_{1, \omega}-\left(\alpha_{1, \omega}+20\right) \bar{\xi}_{\omega}-\delta_{\omega}\left\|\left(\alpha_{1, \omega}+20\right) M^{-1}\right\|_{1} \geq 0, \quad \omega=1,2,3, \\
& 0.3 x_{2}-24-\beta_{2, \omega}-\alpha_{2, \omega} \bar{\xi}_{\omega}-\delta_{\omega}\left\|\alpha_{2, \omega} M^{-1}\right\|_{1} \geq 0, \quad \omega=1,2,3,
\end{array}
$$

or

$$
\begin{array}{ll}
\min & 15 x_{1}+23 x_{2}+\sum_{\omega=1}^{3}\left[\left(-17 \alpha_{1, \omega}-15 \alpha_{2, \omega}\right) \bar{\xi}_{\omega}-17 \beta_{1, \omega}-15 \beta_{2, \omega}\right] \\
\text { st. } \quad & x_{1}+x_{2} \leq 20, \\
& 2.5 x_{1}-\beta_{1, \omega}-\left(\alpha_{1, \omega}+20\right) \bar{\xi}_{\omega}+\delta_{\omega} M^{-1}\left(\alpha_{1, \omega}+20\right) \geq 0, \quad \omega=1,2,3, \\
& 2.5 x_{1}-\beta_{1, \omega}-\left(\alpha_{1, \omega}+20\right) \bar{\xi}_{\omega}-\delta_{\omega} M^{-1}\left(\alpha_{1, \omega}+20\right) \geq 0, \quad \omega=1,2,3, \\
& 0.3 x_{2}-24-\beta_{2, \omega}-\alpha_{2, \omega} \bar{\xi}_{\omega}+\delta_{\omega} M^{-1} \alpha_{2, \omega} \geq 0, \quad \omega=1,2,3, \\
& 0.3 x_{2}-24-\beta_{2, \omega}-\alpha_{2, \omega} \bar{\xi}_{\omega}-\delta_{\omega} M^{-1} \alpha_{2, \omega} \geq 0, \quad \omega=1,2,3 .
\end{array}
$$


Formulation (C.5) if the final form of the robust scenario formulation, which is a LP. When the first-stage decision variables $x_{1}, x_{2}$ are fixed to constant values, (C.5) can be decomposed into 3 subproblems in the following form (for $\omega=1,2,3$ ):

$$
\begin{array}{ll}
\min & \left(-17 \alpha_{1, \omega}-15 \alpha_{2, \omega}\right) \bar{\xi}_{\omega}-17 \beta_{1, \omega}-15 \beta_{2, \omega} \\
\text { st. } & 2.5 x_{1}-\beta_{1, \omega}-\left(\alpha_{1, \omega}+20\right) \bar{\xi}_{\omega}+\delta_{\omega} M^{-1}\left(\alpha_{1, \omega}+20\right) \geq 0, \\
& 2.5 x_{1}-\beta_{1, \omega}-\left(\alpha_{1, \omega}+20\right) \bar{\xi}_{\omega}-\delta_{\omega} M^{-1}\left(\alpha_{1, \omega}+20\right) \geq 0 \\
& 0.3 x_{2}-24-\beta_{2, \omega}-\alpha_{2, \omega} \bar{\xi}_{\omega}+\delta_{\omega} M^{-1} \alpha_{2, \omega} \geq 0 \\
& 0.3 x_{2}-24-\beta_{2, \omega}-\alpha_{2, \omega} \bar{\xi}_{\omega}-\delta_{\omega} M^{-1} \alpha_{2, \omega} \geq 0
\end{array}
$$

The solutions of the 3 subproblems constitute the solution of problem (C.5). 